\title{
THE SPACE OF INCOMPRESSIBLE SURFACES IN A 2-BRIDGE LINK COMPLEMENT
}

\author{
W. FLOYD AND A. HATCHER
}

\begin{abstract}
Projective lamination spaces for 2-bridge link complements are computed explicitly.
\end{abstract}

In this paper we construct a polyhedron $\mathcal{P} \mathcal{L}\left(S^{3}-L_{p / q}\right)$ whose rational points correspond bijectively, in a natural way, with the projective isotopy classes of incompressible surfaces in the exterior of a 2-bridge link $L_{p / q} \subset S^{3}$. Here "projective" means that we factor out by scalar multiplication - taking any number of parallel copies of a surface. (Surfaces are not assumed to be connected.) We expect that $P \mathcal{L}\left(S^{3}-L_{p / q}\right)$ will turn out to be the "projective lamination space of $S^{3}-L_{p / q}$ " as defined and studied for general compact irreducible 3-manifolds in [5 and 12].

An unexpected complication not present in Thurston's theory of projective lamination spaces for surfaces is the fact that $P \mathcal{L}\left(S^{3}-L_{p / q}\right)$ is frequently noncompact, for example for the Whitehead link $L_{3 / 8}$ (see Figure 5.4, upper left-hand corner). However, as in the general theory, $\mathcal{P L}\left(S^{3}-L_{p / q}\right)$ has a natural compactification $\overline{P \mathcal{L}}\left(S^{3}-L_{p / q}\right)$ which is a finite polyhedron.

To construct $P \mathcal{L}\left(S^{3}-L_{p / q}\right)$, we first find a fairly natural finite collection of branched surfaces $B_{i} \subset S^{3}-L_{p / q}$ which carry all the incompressible surfaces in $S^{3}$ $L_{p / q}$. To each $B_{i}$ is associated a convex cell $c_{i}$ whose rational points parametrize the projective classes of surfaces carried by $B_{i}$. Different rational points of $c_{i}$ can determine isotopic surfaces, however, due to the possibility of pushing parts of surfaces across product regions in the complement of $B_{i}$ (the analogue of "digon" regions in the complement of a train track on a surface). This leads to a linear projection $p_{i}: c_{i} \rightarrow \bar{c}_{i}$ of $c_{i}$ onto another convex polyhedral cell $\bar{c}_{i}$, such that over the interior of $\bar{c}_{i}$, projective isotopy classes of surfaces coincide with fibers of $p_{i}$. However, these isotopy relations may not persist over the boundary of $\bar{c}_{i}$. Namely, passing to a face of $\bar{c}_{i}$ corresponds to passing to a branched subsurface of $B_{i}$, and a nonproduct complementary region of this branched subsurface may be decomposed by $B_{i}$ into product complementary regions of $B_{i}$. In this case, rational points of this face of $\bar{c}_{i}$ correspond not to (projective) isotopy classes of incompressible surfaces, but to incompressible surfaces with these limiting "phantom" isotopy relations. The space $\overline{P \mathcal{L}}\left(S^{3}-L_{p / q}\right)$ is formed from the cells $\bar{c}_{i}$ by identifying their faces in the most natural way, distinguishing different "phantom" isotopies between the same sets of surfaces. $\mathcal{P L}\left(S^{3}-L_{p / q}\right)$ consists of the open cells of $\overline{P \mathcal{L}}\left(S^{3}-L_{p / q}\right)$ for which the "phantom" isotopies are actual isotopies.

Received by the editors April 12, 1986.

1980 Mathematics Subject Classification (1985 Revision). Primary 57M25.

Key words and phrases. Incompressible surface, 2-bridge link. 
If $L_{p / q}$ is a knot, i.e., $q$ is odd, then $\mathcal{P} \mathcal{L}\left(S^{3}-L_{p / q}\right)$ is already compact, being a disjoint union of finitely many cubes of various dimensions. This case was essentially done in [6], to which the present paper is something of a sequel.

Though the main emphasis of the paper is on the spaces $P \mathcal{L}\left(S^{3}-L_{p / q}\right)$, our classification of the incompressible surfaces in $S^{3}-L_{p / q}$ allows us to calculate also all the different ways that $S^{3}-L_{p / q}$ fibers over the circle. Here we obtain a geometric "moduli space" for these fiberings, illustrating the general theory in [9].

Some familiarity with [6] on the reader's part will be assumed. For general background on 2-bridge links we refer to [1, 10, and 11].

1. Diagrams of curve systems on the 4-punctured sphere. We first define certain diagrams $D_{t}$ for $t \in[0, \infty]$, which are sketched in Figure 1.1. The diagram $D_{1}$ is the "Diagram of $\mathrm{PSL}_{2}(\mathbf{Z})$ " in [6]. Thus, in the upper half-plane model of the hyperbolic plane $H^{2}, D_{1}$ consists of the (hyperbolic) lines joining pairs $a / b, c / d \in \mathbf{Q} \cup\{\infty\}$ with $a d-b c=1$. These lines subdivide $H^{2}$ into ideal triangles, and $\mathrm{PSL}_{2}(\mathbf{Z})$ is the full group of orientation-preserving combinatorial symmetries of this ideal triangulation of $H^{2}$. Let $G \subset \mathrm{PSL}_{2}(\mathbf{Z})$ be the subgroup of Möbius transformations $(a z+b) /(c z+d)$ with $c$ even. This has index three in $\mathrm{PSL}_{2}(\mathbf{Z})$ and has the ideal triangle $\langle 1 / 0,0 / 1,1 / 1\rangle$ as fundamental domain. The element $z+1 \in G$ identifies the edge $\langle 1 / 0,0 / 1\rangle$ to the edge $\langle 1 / 0,1 / 1\rangle$, while $(z-1) /(2 z-1) \in G$ takes the edge $\langle 0 / 1,1 / 1\rangle$ to itself, reversing its endpoints. Consider the ideal quadrilateral $\langle 1 / 0,0 / 1,1 / 2,1 / 1\rangle$, which is rotated $180^{\circ}$ about its center by $(z-1) /(2 z-1)$. The $G$-images of this quadrilateral form a tiling $Q$ of $H^{2}$ by ideal quadrilaterals. We form the diagram $D_{0}$ from $D_{1}$ by deleting the $G$-orbit of the diagonal $\langle 0 / 1,1 / 1\rangle$ of $\langle 1 / 0,0 / 1,1 / 2,1 / 1\rangle$ and adding the $G$-orbit of the opposite diagonal $\langle 1 / 0,1 / 2\rangle$. Between $D_{0}$ and $D_{1}$ we interpolate a 1-parameter family of $G$-invariant diagrams $D_{t}, 0 \leq t \leq 1$, by inscribing a rectangle in $\langle 1 / 0,0 / 1,1 / 2,1 / 1\rangle$ of monotonically varying shape, as in Figure 1.2, the rectangle collapsing to the diagonals $\langle 1 / 0,1 / 2\rangle$ and $\langle 0 / 1,1 / 1\rangle$ as $t$ approaches 0 and 1 , respectively. Such inscribed rectangles are determined by their vertices, which we take to be the $G$-orbit of a point in the edge $\langle 1 / 0,0 / 1\rangle$, this point moving monotonically from $1 / 0$ to $0 / 1$ as $t$ goes from 0 to 1 . This defines $D_{t}$ for $t \in[0,1]$, and we obtain $D_{t}$ for $t \in[1, \infty]$ by setting $D_{t}=D_{1 / t}$. The edges of $D_{t}$ for $t \neq 0,1, \infty$ fall into four $G$-orbits, labelled $A, B, C, D$ in Figure 1.1. These degenerate into $B$ and $D$ in $D_{0}=D_{\infty}$, and into $A$ and $C$ in $D_{1}$.

The diagrams $D_{t}$ provide a convenient way of parametrizing certain systems of disjoint embedded curves on a 4-punctured sphere, as we shall now explain. The sphere $S^{2}$ we regard as $\mathbf{R}^{2} / \Gamma$, where $\Gamma$ is the group generated by $180^{\circ}$ rotations about points of $\mathbf{Z}^{2}$. The four points of $\mathbf{Z}^{2} / \Gamma$ are viewed as four punctures in $S^{2}$. Pictorially, we shall represent this $S^{2}$ as the plane of the page plus a point at infinity, with the four orbits of $(0,0),(0,1)(1,1)$, and $(1,0)$ forming a square just as these four points do in $\mathbf{R}^{2}$. Figure 1.3 shows the part of $D_{t}$ in the quadrilateral $\langle 1 / 0,0 / 1,1 / 2,1 / 1\rangle$. At the vertices of $D_{t}$ in this quadrilateral are shown certain systems of $\operatorname{arcs}$ on $S^{2}$ with endpoints at the four punctures. These have the property that for a 1- or 2-cell of $D_{t}$ in $\langle 1 / 0,0 / 1,1 / 2,1 / 1\rangle$, the union of the systems of arcs at the vertices of the cell is again a system of arcs in $S^{2}$ meeting only at their endpoints (the punctures). Via the actions of $G \subset \mathrm{PSL}_{2}(\mathbf{Z})$ on $D_{t}$ and on $S^{2}=\mathbf{R}^{2} / \Gamma$, these arc systems on $S^{2}$ corresponding to cells of $D_{t}$ in $\langle 1 / 0,0 / 1,1 / 2,1 / 1\rangle$ extend to arc systems on $S^{2}$ corresponding to all the cells of $D_{t}$. More generally, if we let the 

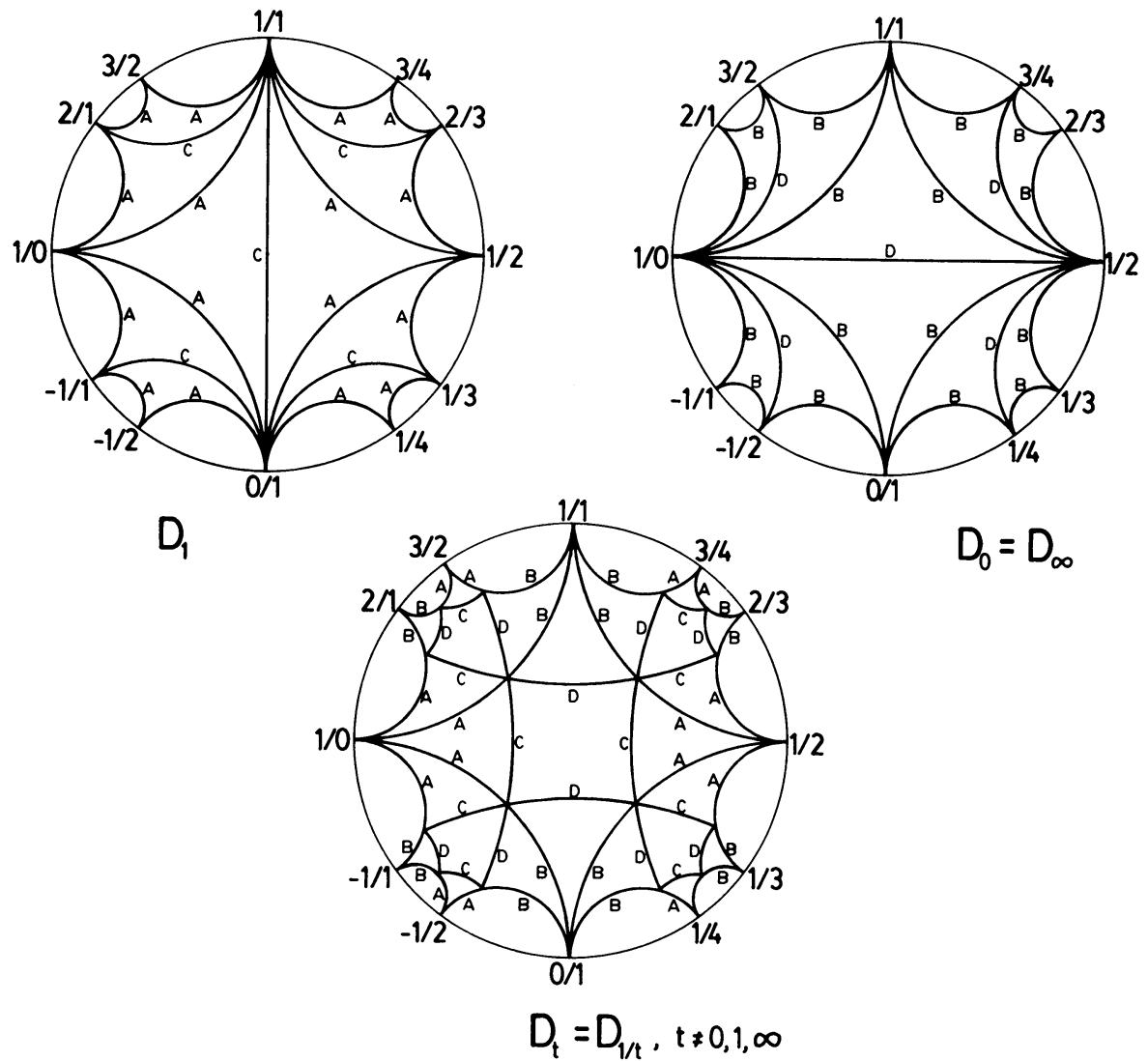

FIGURE 1.1

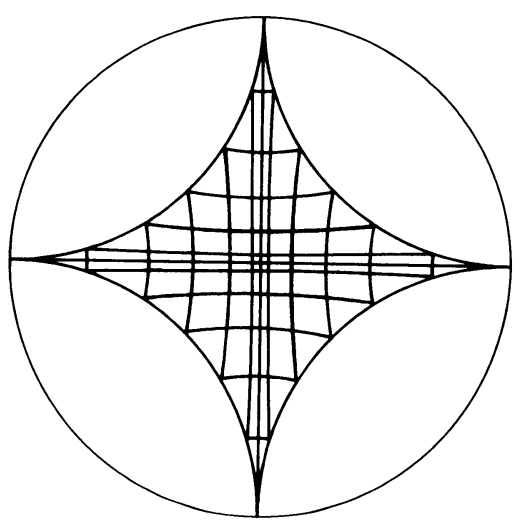

FIGURE 1.2 
labels $a, b, c, \ldots$ in Figure 1.3 be positive integers, and replace an arc labelled $n$ with $n$ nearby parallel copies of itself (with the same endpoints), then we obtain arc systems satisfying:

(1) Each system consists of finitely many arcs which are disjointly embedded except for their endpoints, which lie at the punctures.

(2) No single arc is peripheral, forming a loop bounding a disc in $S^{2}$ whose interior is disjoint from the punctures.

(3) If we call the number of ends of arcs at the puncture $(m, n)$ the incidence number $i(m, n)$, then $i(0,0)=i(0,1) \geq i(1,0)=i(1,1)$.

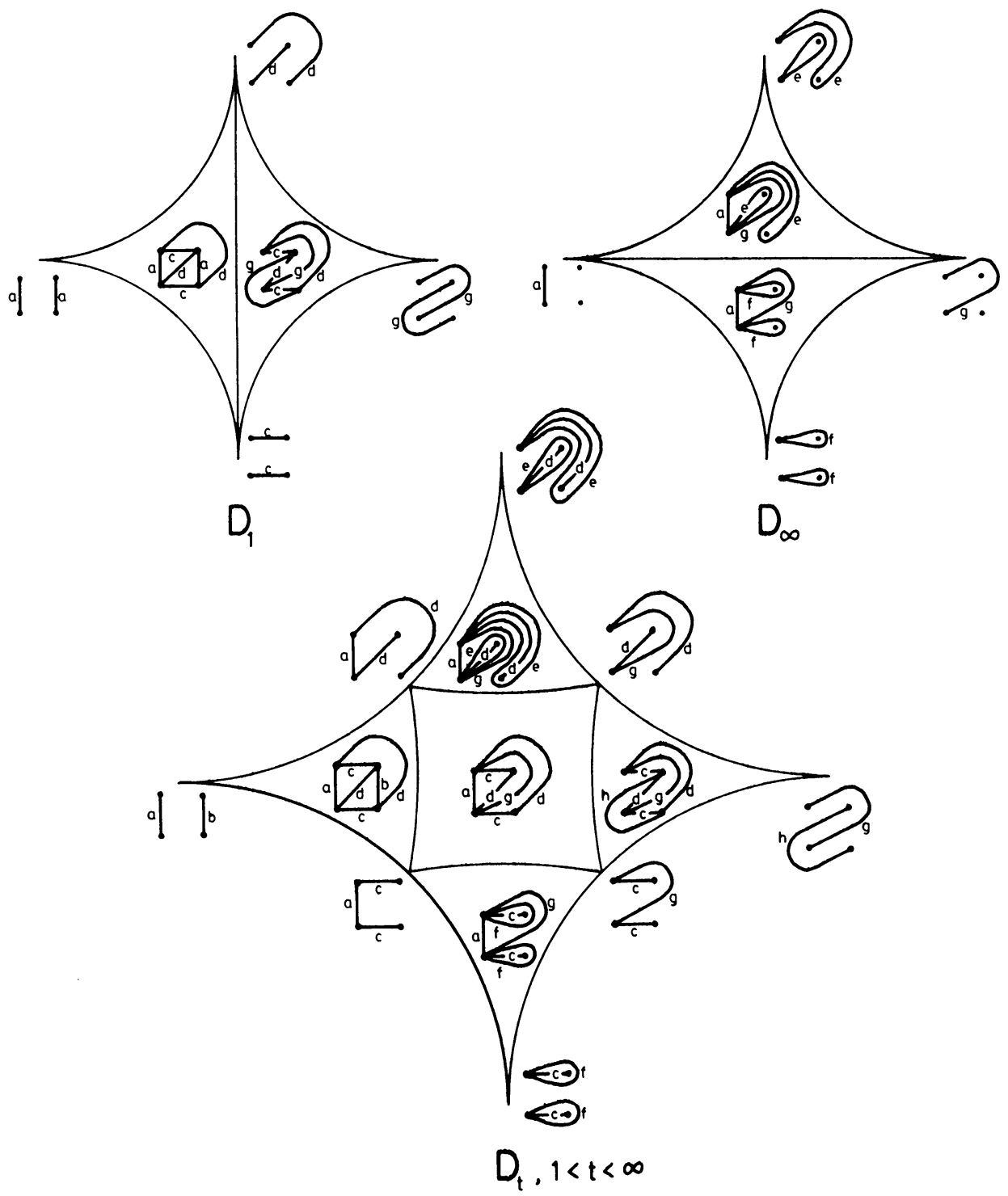

FIGURE 1.3 
Letting $\alpha=i(0,0)=i(0,1)$ and $\beta=i(1,0)=i(1,1)$, we call $\alpha / \beta$ the incidence ratio. Rotation of $S^{2}$ by $180^{\circ}$ about $(1 / 2,1 / 2)$ interchanges the incidence numbers $\alpha$ and $\beta$, so the case $\alpha \leq \beta$ easily reduces to the case $\alpha \geq \beta$ of (3).

With fixed incidence ratio $\alpha / \beta$, the vertices of $D_{\alpha / \beta}$ determine arc systems, unique up to projectivization-identifying an arc system with $n$ parallel copies of itself and hence multiplying all its weights $a, b, c, \ldots$ by $n$. Further, a point of $D_{\alpha / \beta}$ in a 1- or 2-cell which is a rational convex linear combination of the vertices of the cell determines, after multiplying the coefficients by a common denominator, a positive integer linear combination of the vertices, hence an $\operatorname{arc}$ system on $S^{2}$, unique up to projectivization.

PROPOSITION 1.1. Arc systems on the four-punctured sphere satisfying (1)(3) and having a given incidence ratio $\alpha / \beta$, modulo ambient isotopy (fixing the punctures) and projectivization, correspond bijectively with the points of $D_{\alpha / \beta}$ which are rational convex linear combinations of the vertices of $D_{\alpha / \beta}$.

The proof, an exercise in surface topology, is left to the reader.

REMARK. If we drop condition (3), and only specify that the incidence numbers $\alpha, \beta, \gamma, \delta$ at the four punctures have a fixed "ratio" $\alpha: \beta: \gamma: \delta$, then there is a somewhat similar diagram $D_{\alpha: \beta: \gamma: \delta}$ of projective isotopy classes of arc systems in the 4-punctured sphere with the given incidence ratio $\alpha: \beta: \gamma: \delta$. Beside the three cases in Figures 1.1 and 1.3, there turn out to be 28 more such diagrams, which were listed in [2]. These would come into play, for example, if one wanted to classify incompressible surfaces in bundles over the circle with fiber a 4-punctured sphere, using the methods of this paper.

2. Minimal edge-paths. For a given $p / q \in \mathbf{Q}$ with $q$ even we shall be interested in edge-paths from $1 / 0$ to $p / q$ in the diagrams $D_{t}$ which are minimal-no two consecutive edges of the edge-path lying in the same triangle or quadrilateral of $D_{t}$. These minimal edge-paths form in a natural way a 1-complex $\mathcal{E}_{p / q}$. The 0-cells of $\varepsilon_{p / q}$ are the minimal edge-paths in $D_{0}, D_{1}$, and $D_{\infty}$. A minimal edge-path in $D_{t}$ for $t \in(0,1)$ is a point in a 1-cell of $\varepsilon_{p / q}$. As $t$ goes to 0 or 1 , the edge-path in $D_{t}$ approaches an edge-path in $D_{0}$ or $D_{1}$, respectively, which one can easily see is also minimal. These two limiting edge-paths then are the boundary 0-cells of the 1-cell of $\mathcal{E}_{p / q}$. We reason similarly for $t \in(1, \infty)$. Inversion $t \mapsto 1 / t$ induces a bilateral symmetry in $\varepsilon_{p / q}$, since $D_{t}=D_{1 / t}$.

Recall from $\S 1$ the diagram $Q$ of quadrilaterals, which is the intersection of (the 1skeletons of ) all the diagrams $D_{t}$. Dual to $Q$ is a tree $T$, with vertices at the centers of the quadrilaterals of $Q$ and edges joining the centers of adjacent quadrilaterals. There is a unique smallest subtree $T_{p / q} \subset T$ containing vertices corresponding to quadrilaterals of $Q$ containing $1 / 0$ and $p / q$. Dual to $T_{p / q}$ is a finite subcomplex $Q_{p / q}$ of $Q$. Since $T_{p / q}$ contains no branching (i.e., vertices where more than two edges meet), the quadrilaterals of $Q_{p / q}$ are linearly ordered by $T_{p / q}$. It is not hard to see that minimal edge-paths from $1 / 0$ to $p / q$ in the diagrams $D_{t}$ cannot leave $Q_{p / q}$, and must proceed monotonically through the quadrilaterals of $Q_{p / q}$. From this it follows that there are only finitely many minimal edge-paths in $D_{t}$ from $1 / 0$ to $p / q$, i.e., $\mathcal{E}_{p / q}$ is a finite cell complex.

Some examples are given in $\S 5$. 


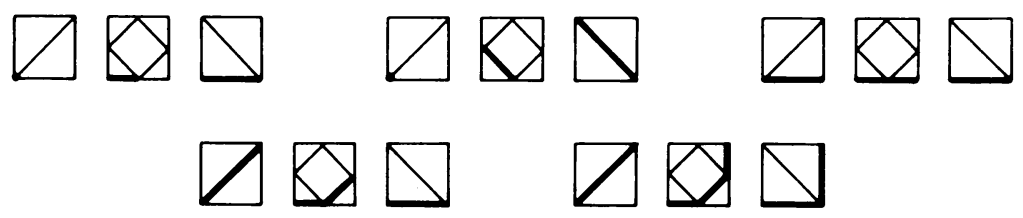

FIGURE 2.1

$\mathcal{E}_{p / q}$ is in fact a simplicial complex: A minimal edge-path in $D_{t}$ with $t \in(0,1)$, say, is determined uniquely by its two limiting edge-paths in $D_{0}$ and $D_{1}$. To see this one can just list all the possibilities for how a minimal edge-path in $D_{t}$ can intersect the part of $D_{t}$ in one quadrilateral of $Q$; up to symmetry there are only five nontrivial intersections, shown in Figure 2.1. In each case the intersections in $D_{0}$ and $D_{1}$ determine the intersection in $D_{t}, t \in(0,1)$.

The following result seems somewhat deeper.

\section{PROPOSITION 2.1. $\mathcal{E}_{p / q}$ is connected (for $q$ even).}

Proof. (The reader may wish to skip this and proceed to §3.) Let $\mathcal{M}(a, x)$ denote the 1-complex of minimal edge-paths in the diagrams $D_{t}, 1 \leq t \leq \infty$, joining the vertices $a$ and $x$, and let $Q(a, x)$ be the minimal subcomplex of $Q$ in which all the edge-paths of $\mathcal{M}(a, x)$ lie. It suffices to show that $\mathcal{M}(a, x)$ is connected. We do this by induction on the number $n$ of quadrilaterals in $Q(a, x)$. The cases $n=1,2$ are trivial, so let us suppose $n \geq 3$. The first two quadrilaterals of $Q(a, x)$ are arranged as in Figure 2.2 (modulo the orientation-reversing symmetry which fixes $a$ and $c$ and interchanges $b$ and $d$ ). The third quadrilateral lies along one of the edges de, ef, or $c f$. Let $\gamma_{1}$ and $\gamma_{2}$ be two vertices of $\mathcal{M}(a, x)$, i.e., minimal edge-paths from $a$ to $x$ in $D_{1}$ or $D_{\infty}$. Suppose first that both $\gamma_{1}$ and $\gamma_{2}$ begin with the edge ad. Let $\gamma_{1}^{\prime}$ and $\gamma_{2}^{\prime}$ be the remaining edges of $\gamma_{1}$ and $\gamma_{2}$. Thus $\gamma_{1}^{\prime}$ and $\gamma_{2}^{\prime}$ represent vertices of $\mathcal{M}(d, x)$. By induction there is a sequence of edge-paths $\gamma^{\prime}$ connecting $\gamma_{1}^{\prime}$ and $\gamma_{2}^{\prime}$ in $\mathcal{M}(d, x)$, each $\gamma^{\prime}$ being an edge-path in $D_{1}, D_{2}$ (say), or $D_{\infty}$. Preceding such a $\gamma^{\prime}$ by the edge $a d$ yields an edge path $\gamma$ from $a$ to $x$, which can fail to be minimal only by going along two sides of a triangle at $d$, entering from the direction of $a$ and leaving toward $c$. This is easily remedied by taking the shorter route across this triangle. It follows that $\gamma_{1}$ and $\gamma_{2}$ lie in the same component of $\mathcal{M}(a, x)$.

It remains to join a vertex $\gamma_{1}$ of $\mathcal{M}(a, x)$ not beginning with $a d$ to a vertex $\gamma_{2}$ which does begin with $a d$. Note that such a $\gamma_{2}$ exists: In $D_{1}$ (or $D_{\infty}$ ) all edge-paths lie in a minimal union $T(a, x)$ of triangles, and $\gamma_{2}$ can be taken as part of the border of $T(a, x)$.

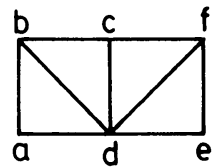

$D_{1}$

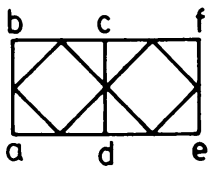

$D_{2}$

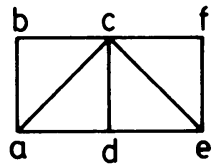

$D_{\infty}$

FIGURE 2.2 
The path $\gamma_{1}$ begins $a b c$ (in $D_{1}$ ) or $a c$ (in $D_{\infty}$ ). Let $\gamma_{1}^{\prime}$ be the rest of $\gamma_{1}$, which is a vertex in $\mathcal{M}(c, x)$.

Case 1. The third quadrilateral of $Q(a, x)$ contains the edge $c f$. Then the quadrilateral $c d e f$ is not part of $Q(c, x)$, so we can compose minimal edge-paths from $a$ to $c$ with minimal edge-paths from $c$ to $x$ and the result will automatically be minimal. In particular, given the simple structure of $\mathcal{M}(a, c)$ and the inductive hypothesis that $\mathcal{M}(c, x)$ is connected (and has at least two vertices), we see that if $\gamma_{1}$ begins $a b c$, there is a 1-simplex in $\mathcal{M}(a, x)$ joining $\gamma_{1}$ to a path beginning $a c$, and similarly if $\gamma_{1}$ begins ac there is a 1-simplex in $\mathcal{M}(a, x)$ joining $\gamma_{1}$ to a path $\gamma_{2}$ beginning $a d$.

Case 2. The third quadrilateral of $Q(a, x)$ contains de or $e f$. The quadrilateral cdef is then in $Q(c, x)$, so there exists a vertex $\gamma_{2}^{\prime}$ of $\mathcal{M}(c, x)$ beginning $c d$. By induction, there is a sequence of edge-paths $\gamma^{\prime}$ connecting $\gamma_{1}^{\prime}$ and $\gamma_{2}^{\prime}$ in $\mathcal{M}(c, x)$. For such a $\gamma^{\prime}$, let $\gamma^{\prime \prime}$ be $\gamma^{\prime}$ minus any initial segment in $c d$. We can precede $\gamma^{\prime \prime}$ by an edge-path in $a b c d$ to form a minimal edge-path $\gamma$ from $a$ to $x$. Specifically, use a path inside the triangle $a b c$ unless $\gamma^{\prime}$ starts down $c d$. Minimality then determines $\gamma$ uniquely. These $\gamma$ 's give a way of connecting $\gamma_{1}$ to a vertex $\gamma_{2}$ in $\mathcal{M}(a, x)$, which passes through $d$ by the choice of $\gamma_{2}^{\prime}$.

3. Branched surfaces for 2-bridge link complements. It will be convenient to expand $\mathcal{E}_{p / q}$ slightly to another 1-complex $\tilde{\mathcal{E}}_{p / q}$ by replacing each vertex $v_{\gamma}$ of $\varepsilon_{p / q}$ corresponding to a minimal edge-path $\gamma$ in $D_{1}$ containing $C$-type edges by a 1-simplex $e_{\gamma}$. The 1-cells of $\varepsilon_{p / q}$ attaching to $v_{\gamma}$ which correspond to minimal edgepaths in $D_{t}$ with $t \in(0,1)$ become 1-cells in $\tilde{\mathcal{E}}_{p / q}$ attaching to one end of $e_{\gamma}$, while at the other end of $e_{\gamma}$ are attached the 1-cells of $\varepsilon_{p / q}$ abutting $v_{\gamma}$ which correspond to minimal edge-paths in $D_{t}$ with $t \in(1, \infty)$. Thus $\tilde{\mathcal{E}}_{p / q}$ has an involution induced by $t \mapsto 1 / t$, which reverses the ends of the new 1 -simplices $e_{\gamma}$.

Let $L_{p / q} \subset S^{3}$ be a 2-bridge link of two components, i.e., with $q$ even. Our immediate goal, in the next few paragraphs, is to construct for each 0- or 1-cell $c$ of $\tilde{\mathcal{E}}_{p / q}$ a branched surface $\Sigma_{c}$ in $S^{3}-L_{p / q}$. As in [6] we regard $S^{3}$ as the 2-point compactification of $S^{2} \times \mathbf{R}$, and position $L_{p / q}$ in a shell $S^{2} \times I$ so that it meets $S^{2} \times 0$ and $S^{2} \times 1$ each in two arcs, and each intermediate level $S_{r}^{2}=S^{2} \times r$ in four points. As in $\S 1$, we parametrize $S_{r}^{2}$ as the quotient $\mathbf{R}^{2} / \Gamma$, where $\Gamma$ is the group generated by $180^{\circ}$ rotations of $\mathbf{R}^{2}$ about points of $\mathbf{Z}^{2}$. The four points of $\mathbf{Z}^{2} / \Gamma$ correspond to the four points of $L_{p / q} \cap S_{r}^{2}$; the two arcs of $L_{p / q} \cap S_{0}^{2}$ have slope $1 / 0$, and those of $L_{p / q} \cap S_{1}^{2}$ have slope $p / q$. $\operatorname{PSL}_{2}(\mathrm{Z})$ acts linearly on each level $S_{r}^{2}=\mathbf{R}^{2} / \Gamma$, leaving $\mathbf{Z}^{2} / \Gamma$ invariant.

Consider first a 1-cell $c$ of $\tilde{\mathcal{E}}_{p / q}$ corresponding to an edge-path $\gamma$ in $D_{t}$ with $t \in(1, \infty)$. Let $e_{1}, \ldots, e_{k}$ be the sequence of edges of $\gamma$. Each $e_{i}$ will determine a branched surface $\Sigma_{e_{i}}$ in $S^{2} \times[(i-1) / k, i / k]$ and the desired branched surface $\Sigma_{c}$ will be the union of these $\Sigma_{e_{i}}$ 's. The four classes $A, B, C, D$ of edges of $D_{t}$ determine four corresponding types of branched surfaces $\Sigma_{A}, \Sigma_{B}, \Sigma_{C}$, and $\Sigma_{D}$. For the $A$ and $B$ edges in the line $\langle 1 / 0,0 / 1\rangle$ and the $C$ and $D$ edges abutting this line from above, these branched surfaces are shown in Figure 3.1.

In each picture, the top of the branched surface consists of one arc of slope $\infty$ and two arcs of slope 0 . This configuration corresponds to the vertex of $D_{t}$ in $\langle 1 / 0,0 / 1\rangle$. At the bottom of the branched surface there are arcs of slope $\infty$ for $\Sigma_{A}, 0$ for $\Sigma_{B}$, 

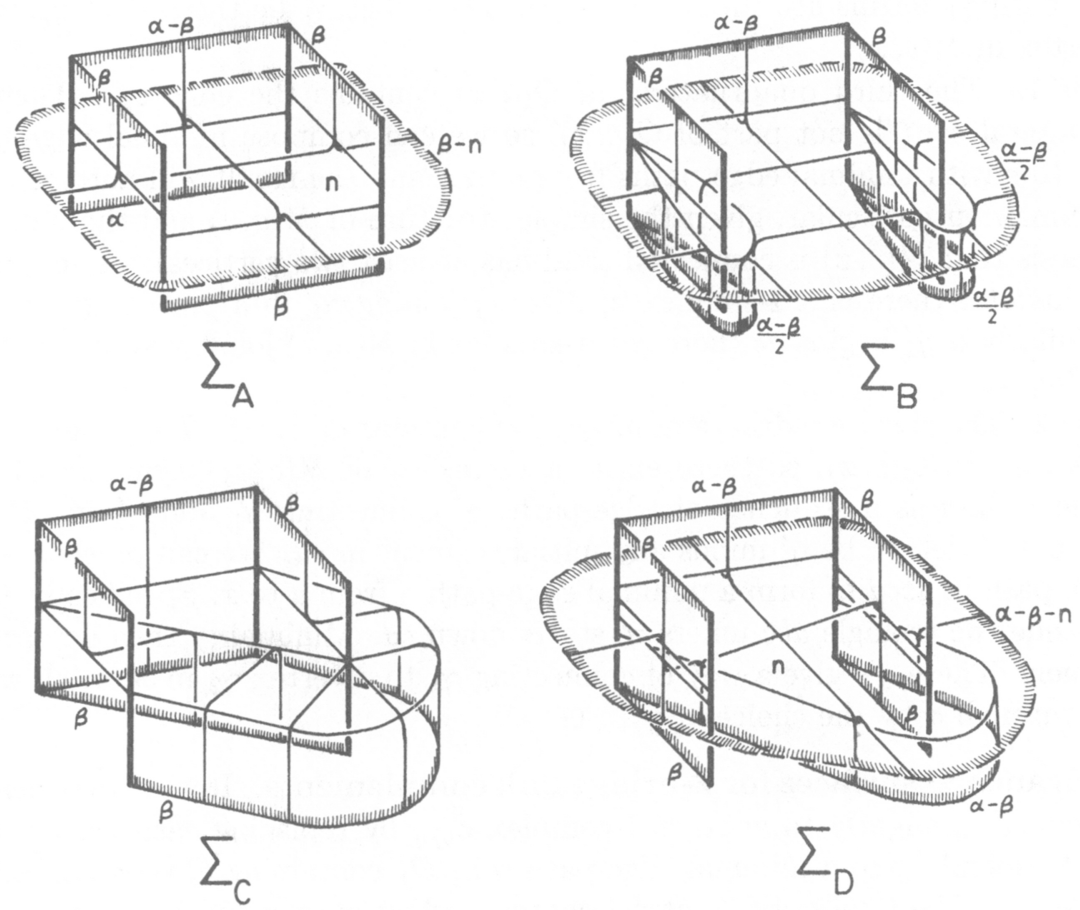

FIGURE 3.1

$\infty$ and 1 for $\Sigma_{C}$, and 0 and $1 / 2$ for $\Sigma_{D}$, corresponding to the other endpoints of these fcur edges $A, B, C$, and $D$. The four vertical arcs of each branched surface are on $L_{p / q}$. An edge $e_{i}$ of $\gamma$ is the image of one of these four edges under a unique $g_{i} \in G$. To get $\Sigma_{e_{i}}$ we first apply $g_{i} \times \operatorname{id}_{I}$ to the appropriate surface $\Sigma_{A}, \Sigma_{B}, \Sigma_{C}$, or $\Sigma_{D}$, and then scale vertically into the interval $[(i-1) / k, i / k]$, reflecting through a horizontal $S^{2}$ if $e_{i}$ has the opposite orientation from $g_{i}(A), g_{i}(B), g_{i}(C)$, or $g_{i}(D)$.

This defines $\Sigma_{c}$ for $c$ an edge of $\tilde{\mathcal{E}}_{p / q}$ corresponding to the edge-path $\gamma$ in $D_{\alpha / \beta}$ with $0<\beta<\alpha$. Letting $\beta$ go to zero, $\gamma$ becomes a minimal edge-path $\gamma_{\infty}$ in $D_{\infty}$, corresponding to a 0 -cell $c_{\infty}$ of $\tilde{\mathcal{E}}_{p / q}$. For such a $\gamma_{\infty}$ we construct $\Sigma_{c_{\infty}}$ by the same procedure as above using only the branched surfaces $\Sigma_{B}^{\infty} \subset \Sigma_{B}$ and $\Sigma_{D}^{\infty} \subset \Sigma_{D}$ obtained by deleting the sheets labelled $\beta$. Deleting $\beta$ sheets from $\Sigma_{A}$ or $\Sigma_{C}$ segments leaves only vertical rectangles, which can be absorbed into the adjacent segments. So an inclusion $c_{\infty} \subset \partial c$ gives rise to an inclusion $\Sigma_{c_{\infty}} \subset \Sigma_{c}$ (up to isotopy in the $I$ factor of $S^{2} \times I$ ).

Similarly, when $\alpha-\beta$ goes to zero, $\gamma$ becomes a minimal edge-path $\gamma_{1}$ in $D_{1}$ corresponding to the 0-cell $c_{1} \in \tilde{\mathcal{E}}_{p / q}$ at the other end of $c$. For this $c_{1}, \Sigma_{c_{1}} \subset \Sigma_{c}$ is built from the segments $\Sigma_{A}^{1} \subset \Sigma_{A}$ and $\Sigma_{C}^{1} \subset \Sigma_{C}$ obtained by deleting sheets labelled $\alpha-\beta$, and each such inclusion $c_{1} \subset \partial c$ gives rise to an inclusion $\Sigma_{c_{1}} \subset \Sigma_{c}$ (up to isotopy). If $\gamma_{1}$ contains $C$-type edges, we can enlarge $\Sigma_{c_{1}}$ to a branched surface $\Sigma_{c}$, where $c$ is the 1-cell of $\tilde{\mathcal{E}}_{p / q}$ corresponding to $\gamma_{1}$, by enlarging the $\Sigma_{C}^{1}$ segments of $\Sigma_{c_{1}}$ to the $\Sigma_{A}^{1}$ segments which contain them; this is possible since 
$\alpha-\beta=0$. (Recall that $D_{1}$ has the full group $\mathrm{PSL}_{2}(\mathrm{Z})$ as symmetry group, which identifies $A$ and $C$ edges.)

This covers the cases $1 \leq \alpha / \beta \leq \infty$. The other cases are obtained by rotating $S^{2}=\mathbf{R}^{2} / \Gamma$ by $180^{\circ}$ about the point $(1 / 2,1 / 2)$, interchanging the components of $L_{p / q}$ and hence inverting $\alpha / \beta$.

A surface carried by one of the branched surfaces $\Sigma_{c}$ is determined by $\alpha$ and $\beta$, the numbers of sheets of the surface running along each component of $L_{p / q}$, and by how the surface branches in each segment $\Sigma_{A}, \Sigma_{B}, \Sigma_{C}$, or $\Sigma_{D}$ of $\Sigma_{c}$. For a $\Sigma_{C}$ segment there is no branching. For a $\Sigma_{B}$ segment the branching is uniquely determined: half of the $\alpha-\beta$ parallel sheets go each way. For a $\Sigma_{A}$ segment the branching is specified by the numbers, $n$ and $\beta-n(0 \leq n \leq \beta)$, of sheets near each of the two saddles of $\Sigma_{A}$. For a $\Sigma_{D}$ segment the branching is determined by the numbers, $n$ and $\alpha-\beta-n(0 \leq n \leq \alpha-\beta)$, of sheets in each of the two horizontal unbranched parts of $\Sigma_{D}$.

For the sake of simplicity we shall restrict our attention to incompressible surfaces $S \subset S^{3}-L_{p / q}$ satisfying also the following natural "meridional incompressibility" condition:

If there is a disc $D \subset S^{3}$ with $D \cap S=\partial D$ and $D$ meeting $L_{p / q}$ transversely in one point in the interior of $D$, then there is a disc $D^{\prime} \subset S \cup L_{p / q}$ with $\partial D^{\prime}=\partial D, D^{\prime}$ also meeting $L_{p / q}$ transversely in one interior point.

This condition holds automatically if $\alpha \beta \neq 0$, since no such $D$ can exist then. A surface $S \subset S^{3}-L_{p / q}$ not satisfying (*) can be "meridionally surgered" repeatedly until a surface which does satisfy $(*)$ results. It is easy to check that such meridional surgery always preserves incompressibility. However, the inverse "meridional tubing" operation need not preserve incompressibility. We shall return to this question in $\S 8$.

Here is the main result, which will be the basis for the construction of the polyhedron $P \mathcal{L}\left(S^{3}-L_{p / q}\right)$ in the next section.

THEOREM 3.1. (a) The orientable incompressible surfaces in $S^{3}-L_{p / q}$, without peripheral components and satisfying (*) above, are exactly (up to isotopy) the orientable surfaces carried by the branched surfaces $\Sigma_{c}$ as $c$ ranges over the 1-cells of $\tilde{\mathcal{E}}_{p / q}$.

(b) If two branched surfaces $\Sigma_{c}$ and $\Sigma_{c^{\prime}}$ corresponding to different 1-cells $c$ and $c^{\prime}$ of $\tilde{\mathcal{E}}_{p / q}$ carry isotopic surfaces $S$ and $S^{\prime}$ then $c$ and $c^{\prime}$ have a common endpoint $e$ and the branched surface $\Sigma_{e}$ carries a surface isotopic to $S$ and $S^{\prime}$.

(c) For c a 0 - or 1-cell of $\tilde{\mathcal{E}}_{p / q}$, corresponding to the edge-path $\gamma$ in $D_{t}$, the relation of isotopy among the surfaces carried by $\Sigma_{c}$ is generated by.

(i) Different branchings in an initial or final $A$ - or D-type segment of $\Sigma_{c}$ yield isotopic surfaces.

(ii) If two successive edges $e_{i}$ and $e_{i+1}$ of $\gamma$ are $A$ - or $D$-type edges separated by only one edge in $D_{t}$, then the branching data in Figure 3.2 yield isotopic surfaces.

REMARK. As will be observed later, the isotopy relations (i) and (ii) arise from pushing across $I$-bundle components of the complement of $\Sigma_{c}$ in $S^{3}-L_{p / q}$. Compare [7]. 


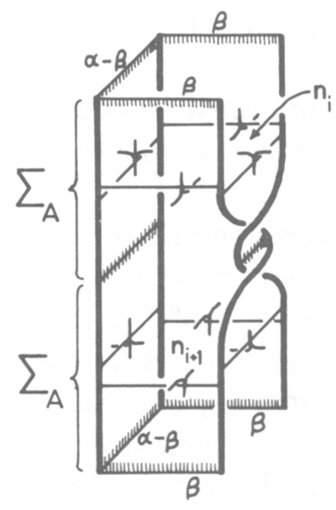

$\left(n_{i}, n_{i+1}\right) \approx\left(n_{i}+1, n_{i+1}+1\right)$

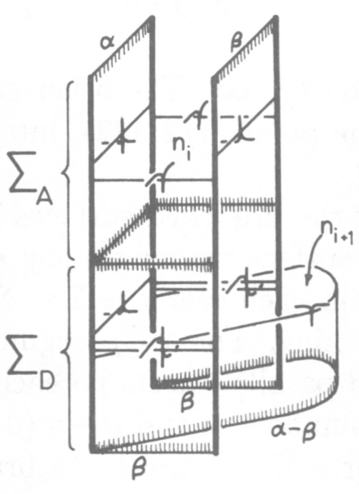

$\left(n_{i}, n_{i+1}\right) \approx\left(n_{i}+1, n_{i+1}-1\right)$

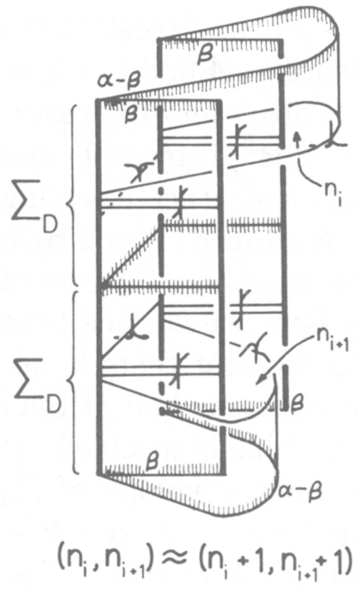

FIGURE 3.2

4. The polyhedron $P \mathcal{L}_{p / q}$. This will be a subpolyhedron of $\mathcal{P} \mathcal{L}\left(S^{3}-L_{p / q}\right)$ whose rational points correspond to incompressible surfaces satisfying the meridional incompressibility condition (*). Enlarging $P \mathcal{L}_{p / q}$ to $\mathcal{P} \mathcal{L}\left(S^{3}-L_{p / q}\right)$ does not add much of interest, and will be described rather briefly in $\S 8$.

Consider a branched surface $\Sigma_{c}$ for $c$ a 1-cell of $\tilde{\varepsilon}_{p / q}$ corresponding to a minimal edge-path $\gamma$ in $D_{t}, 1<t<\infty$. Fixing the numbers $\alpha$ and $\beta$ of sheets at each component of $L_{p / q}, 0<\beta<\alpha$, the surfaces carried by $\Sigma_{c}$ are determined by how they branch in the $\Sigma_{A}$ and $\Sigma_{D}$ segments of $\Sigma_{c}$. As explained in $\S 3$, for a $\Sigma_{A}$ segment the choice is determined by an integer $n \in[0, \beta]$, while for a $\Sigma_{D}$ segment the choice is determined by an integer $n \in[0, \alpha-\beta]$. Projectivizing by identifying a surface with any number of parallel copies of itself, only the ratio $\alpha / \beta$ matters, and the branching numbers $n$ become rational numbers in an interval. So for fixed $\alpha / \beta$, the projective classes of surfaces carried by $\Sigma_{c}$ correspond to the rational points of a cube $I^{a} \times I^{d}$, where $a$ is the number of $\Sigma_{A}$ segments of $\Sigma_{c}$ and $d$ is the number of $\Sigma_{D}$ segments. Letting $\alpha / \beta$ range from 1 to $\infty$, the $I^{d}$ factor collapses to a point when $\alpha / \beta=1$ and the $I^{a}$ factor collapses to a point when $\alpha / \beta=\infty$. So all the projective classes of surfaces carried by $\Sigma_{c}$ are parametrized by the rational points of the join $I^{a} * I^{d}$. The two ends $I^{a}$ and $I^{d}$ of this join parametrize the surfaces carried by the two branched subsurfaces $\Sigma_{c_{1}}$ and $\Sigma_{c_{\infty}}$ corresponding to the two endpoints $c_{1}$ and $c_{\infty}$ of the 1-cell $c$.

In similar fashion, the projective classes of surfaces carried by a branched surface $\Sigma_{c}$, with $c$ a 1 -cell of $\tilde{\mathcal{E}}_{p / q}$ corresponding to a minimal edge-path $\gamma_{1}$ in $D_{1}$ containing $C$-type edges, are parametrized by the rational points of a cube $I^{a+c}$, where $a$ and $c$ are the numbers of $A$ - and $C$-type edges of $\gamma_{1}$. If $c_{\infty}$ is the endpoint of $c$ in $\tilde{\mathcal{E}}_{p / q}$ on the $\infty$ side, the inclusion $\Sigma_{c_{\infty}} \subset \Sigma_{c}$ corresponds to an inclusion $I^{a} \subset I^{a+c}=I^{a} \times I^{c}$ in which $\left(n_{1}, \ldots, n_{a}\right)$ goes to $\left(n_{1}, \ldots, n_{a}, \varepsilon_{1}, \ldots, \varepsilon_{c}\right)$, with each $\varepsilon_{i}$ either 0 or 1 .

The cases $\alpha \leq \beta$ are similar, but we note that the $180^{\circ}$ rotation of $S^{2}=\mathbf{R}^{2} / \Gamma$ about $(1 / 2,1 / 2)$ which interchanges the two components of $L_{p / q}$, hence interchanges $\alpha$ and $\beta$, has the effect of flipping the $I$ factors of the cubes $I^{c}$ end for end (see the picture of $\Sigma_{C}$ in Figure 3.1), hence changing the inclusions $I^{a} \subset I^{a} \times I^{c}$ in 
the preceding paragraph to their "opposite" inclusions, i.e., interchanging the cases $\varepsilon_{i}=0$ and $\varepsilon_{i}=1$.

From the disjoint union of these various cubes $I^{a}, I^{a+c}$, and $I^{d}$ and joins $I^{a} * I^{d}$ corresponding to the cells of $\tilde{\mathcal{E}}_{p / q}$ we can form a finite polyhedron $\chi_{p / q}$ by identifying via the inclusions corresponding to the way the 1-cells of $\tilde{\varepsilon}_{p / q}$ attach to the 0-cells. However, $x_{p / q}$ is not yet the polyhedron $P \mathcal{L}_{p / q}$ we seek, because we have not yet taken into account the isotopy relations which hold among the surfaces carried by one of our branched surfaces (specified in Theorem 3.1(c)).

Again let us fix $\alpha / \beta$ with $1<\alpha / \beta<\infty$ and consider a $\Sigma_{c}$ corresponding to a minimal edge-path $\gamma$ in $D_{\alpha / \beta}$. A maximal sequence of consecutive $A$ - and $D$-type edges of $\gamma$, each separated from the next by only one edge in $D_{\alpha / \beta}$, as in Theorem 3.1(c)(ii), we call a string. (A string containing only one edge is permitted.) A string of $l$ edges contributes a factor $I^{l}$ to the $\alpha / \beta$-slice $I^{a} \times I^{d}$ of the join $I^{a} * I^{d}$ in $X_{p / q}$ corresponding to $\Sigma_{c}$. We may parametrize these $l I$ factors so that each of the three types of isotopy relations in (c)(ii) takes the algebraic form $\left(n_{1}, n_{i+1}\right) \sim$ $\left(n_{i}+1, n_{i+1}-1\right)$, so that the sum $\sum_{i=1}^{\infty} n_{i}$ is an invariant of these three isotopy relations. It is easy to see that in fact $\sum_{i=1}^{l} n_{i}$ is the only invariant of these three isotopy relations. (Think of a row of $l$ stacks of coins, the $i$ th stack having $n_{i}$ coins, $n_{i}$ being subject to the restraint $0 \leq n_{i} \leq N_{i}$ for fixed $N_{i} \geq 1$. By repeated operations of transferring a coin from one stack to an adjacent (nonfull) stack we can go from any one admissible distribution of $\sum_{i=1}^{l} n_{i}$ coins to any other. Namely, we can fill up the first $m$ stacks, partially fill the $(m+1)$ th stack, and have the remaining $l-m-1$ stacks empty, for some $m$.) Thus after projectivizing, the isotopy relations of (c)(ii) induce an equivalence relation on the cube $I^{l}$ resulting in a quotient space which is an interval. Moreover, if the string in question occurs at the beginning or end of the edge-path $\gamma$, this interval quotient of $I^{l}$ is further collapsed to a point by isotopy relation (c)(i).

Summarizing, the projective isotopy classes of surfaces carried by $\Sigma_{c}$ with fixed $\alpha / \beta, 1<\alpha / \beta<\infty$, correspond bijectively with the rational points of a cube whose dimension is the number of strings of $\gamma$ which are not at the beginning or end of $\gamma$. (Note that by Figure 1.1, $\gamma$ starts and ends with $A$-type edges, hence with strings. Possibly $\gamma$ is itself entirely a single string; this special situation has geometric consequences for $S^{3}-L_{p / q}$, as will be described in §6.)

The same analysis applies to other fixed values of $\alpha / \beta$. Letting $\alpha / \beta$ vary, however, we see an interesting phenomenon: A string in a minimal edge-path $\gamma$ in $D_{\alpha / \beta}$, with $\alpha / \beta \neq 0,1, \infty$, can break up into several strings in the limiting edge-paths $\gamma_{0}$, $\gamma_{1}$, or $\gamma_{\infty}$ as $\alpha / \beta$ goes to 0,1 , or $\infty$. This phenomenon can be analyzed as follows.

The $D$-type edges in the diagram $D_{0}\left(=D_{\infty}\right)$ form a tree with vertices the fractions $p / q$ with $q$ even. So there is a unique nonretracing path $\gamma_{p / q}$ in this tree from $1 / 0$ to such a $p / q$. If $e_{0}, \ldots, e_{k}$ are the edges of $\gamma_{p / q}$, in order, then $\gamma_{p / q}$ defines and is defined by a sequence $d_{1}, \ldots, d_{k}$ of nonzero integers, where $e_{i}$ is the $\left|d_{i}\right|$ th $D$-edge to the right (if $d_{i}<0$ ) or left (if $d_{i}>0$ ) of $e_{i-1}$. Moreover, the configuration $Q_{p / q}$ of quadrilaterals defined in $\S 2$ consists of the $k+1$ quadrilaterals having $e_{0}, \ldots, e_{k}$ as diagonals plus, for each $i \in[1, k]$, the $\left|d_{i}\right|-1$ quadrilaterals containing the $D$-edges between $e_{i-1}$ and $e_{i}$. The possibilities for how a string of $A$ - and $D$-edges of $D_{\alpha / \beta}, \alpha / \beta \neq 0,1, \infty$, passes through the portion of $Q_{p / q}$ from $e_{i-1}$ to $e_{i}$ are shown by the heavy lines in Figure 4.1. (In the case $d_{i}= \pm 2$, the two 

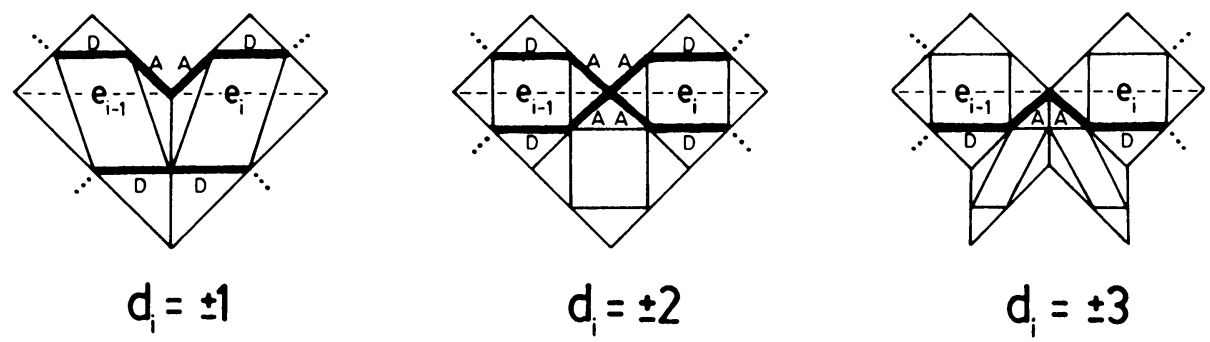

FIGURE 4.1

paths cross at the center point.) Thus as $\alpha / \beta$ goes to 0 or $\infty$, the string breaks between successive $A$-edges meeting at a vertex where $d_{i}= \pm 2$ or \pm 3 , while as $\alpha / \beta$ goes to 1 , the string breaks between successive $D$-edges where $d_{i}= \pm 1$. Also, it is easy to see from these pictures that nothing else besides such breakage, for example, amalgamation of strings, occurs as $\alpha / \beta$ goes to a limiting value of 0,1 , or $\infty$.

The effect of this string-breaking phenomenon is that the natural quotient space of $\chi_{p / q}$ obtained by collapsing cube slices with fixed $\alpha / \beta$ to quotient cubes according to the isotopy relations of Theorem 3.1(c), as described above, has in general a nonHausdorff topology, the equivalence relations becoming finer in the limit. As we want $P \mathcal{L}_{p / q}$ to be Hausdorff, in fact a polyhedron, we proceed as follows.

The cubes $I^{a}, I^{a+c}$, and $I^{d}$ corresponding to cells $c$ of $\tilde{\mathcal{E}}_{p / q}$ have quotient cells (cubes) $I^{a} / c, I^{a+c} / c$, and $I^{d} / c$ determined by their isotopy relations. A join $I^{a} * I^{d}$, corresponding to a 1-cell $c$ of $\tilde{\mathcal{E}}_{p / q}$, has the equivalence relation given by isotopy on its slices $I^{a} \times I^{d}$ (corresponding to fixed $\alpha / \beta$ ). By continuity this relation extends over the ends $I^{a}$ and $I^{d}$ of $I^{a} * I^{d}$. Let $I^{a} * I^{d} / c$ denote the quotient by this extended relation; this is a cell, with a natural linear structure. It has two end faces which are the limiting quotient cells of $I^{a}$ and $I^{d}$. These limiting cells are indexed by vertices of $\tilde{\mathcal{E}}_{p / q}$ together with induced limiting decompositions into strings of the corresponding edge-paths in $D_{0}, D_{1}$, or $D_{\infty}$, decompositions which may be coarser than the intrinsic string decompositions. (An induced limiting string decomposition specifies how to form the limiting quotient cell of $I^{a}$ or $I^{d}$.)

The finite polyhedron $\overline{\mathcal{P L}_{p / q}}$ is obtained by taking all the quotient cells $I^{a} / c, I^{d} / c$, $I^{a+c} / c$, and $I^{a} * I^{d} / c$, which correspond bijectively with cells $c$ of $\tilde{\mathcal{E}}_{p / q}$, and making all the natural face identifications. Namely, the cells $I^{a+c} / c$ have certain cells $I^{a} / c$ as faces, and the cells $I^{a} * I^{d} / c$ have as faces either certain $I^{a} / c$ or $I^{d} / c$ cells, or quotient cells of these corresponding to coarser limiting string decompositions, as described above. Throwing out these latter cells we obtain the subspace $P \mathcal{L}_{p / q}$. This inherits a polyhedral structure from $\overline{P \mathcal{L}}_{p / q}$, since it is the complement of a subpolyhedron of $\overline{\mathcal{P L}}_{p / q}$.

The possibilities for limiting quotient cells are well illustrated in the examples of $\S 5$ below. A good example is the vertex " 4 " for $p / q=[4,3,4]$, where the quotient cell is $I^{2}$ but there are also two limiting intervals and a limiting vertex. In the case $p / q=[4,2,4]$, the vertex " 4 " gives a quotient cell $I$ together with three different limiting vertices. 
5. Examples. Generally speaking, the complexity of $P \mathcal{L}_{p / q}$ increases with the complexity of the (positive) continued fraction expansion of $p / q$. We shall describe here three of the simplest families
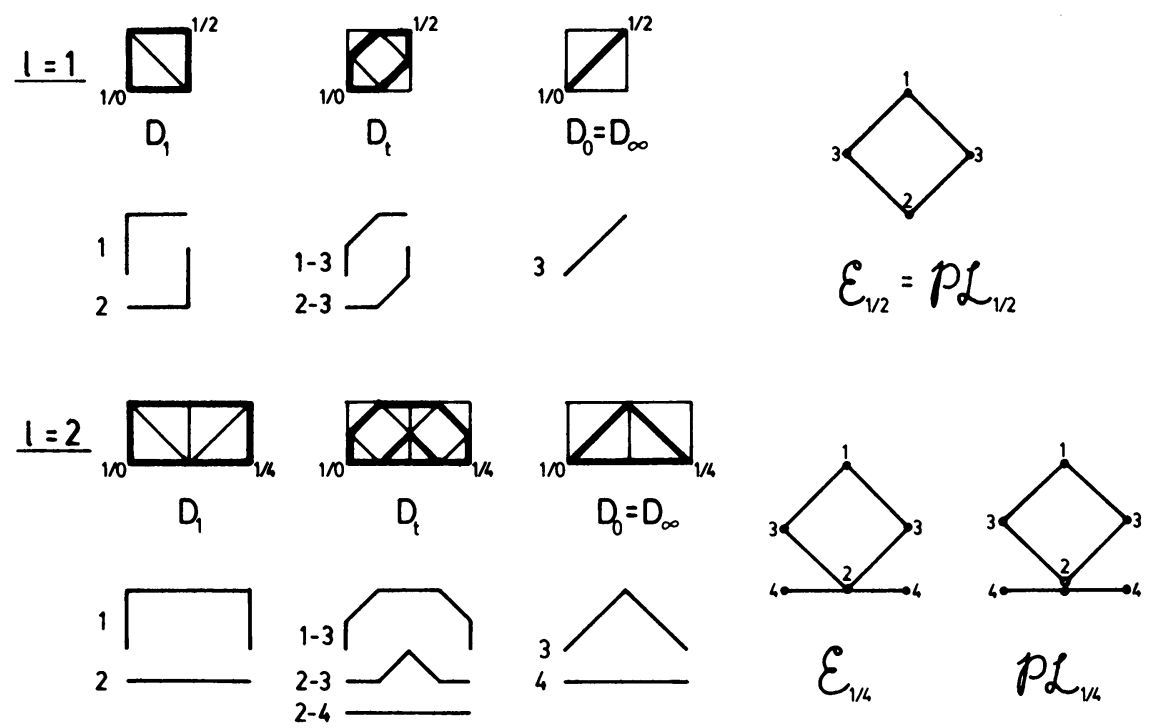

FIGURE 5.1
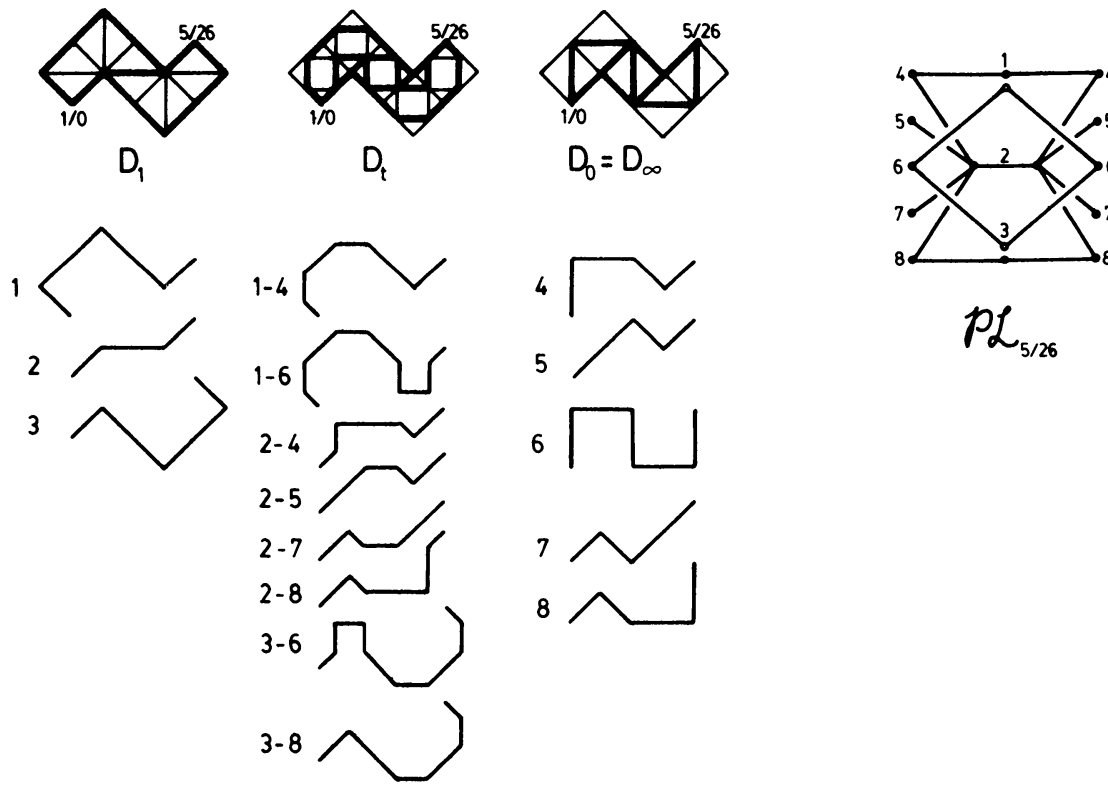

FIGURE 5.2 
(I) $p / q=[2 l]=1 / 2 l$. The cases $l=1,2$ are shown in Figure 5.1. For $l>2$, $P \mathcal{L}_{1 / 2 l}$ is identical with $P \mathcal{L}_{1 / 4}$. The unshaded vertex belongs to $\overline{P \mathcal{L}}-P \mathcal{L}$. (We continue this shading convention in the following examples.) It arises from a string breaking, as in $\S 4$.

(II) $p / q=[2 l+1,2 m+1]=1 /(2 l+1)+1 /(2 m+1)$. The generic case, shown in Figure 5.2, is $l=m=2$, hence $p / q=5 / 26$. Increasing $l$ or $m$ does not affect $P \mathcal{L}_{p / q}$. If $l=1$, the only change is to delete the two vertices " 5 " (and the edges leading to them). If $m=1$, the two vertices "7" are deleted.

(III) $p / q=[2 l, m, 2 n]=1 / 2 l+1 / m+1 / 2 n$. Here the generic case is $p / q=$ $[4,3,4]=13 / 56$. Figure 5.3 shows the calculation of $\mathcal{E}_{13 / 56}$. Some vertices are deleted with smaller values of $l, m$, and $n$ : " 4 " if $m=1$, "7" and "9" if $l=1$, " 8 " and " 9 " if $n=1$. The polyhedra $P \mathcal{L}_{p / q}$ for $p / q=[2 l, m, 2 n]$ are shown in Figure 5.4. These nine possibilities cover all cases (up to interchange of $l$ and $n$ ), since increasing $2 l$ or $2 n$ beyond 4 or $m$ beyond 3 has no effect on $P \mathcal{L}_{p / q}$. We note the "non-Hausdorff" behavior which occurs for example for $p / q=[4,2,2]$, where the edges labelled 2-6 appear to approach the whole edge labelled 2, but in reality approach the new unshaded vertex 2 . In the generic case $p / q=[4,3,4]$ there are two unshaded edges labelled 4 (corresponding to the two projections of the square 4 onto its factors) in addition to the unshaded vertex labelled 4.

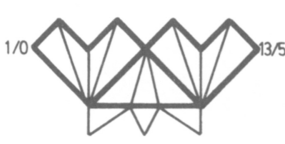

$\mathrm{D}_{1}$
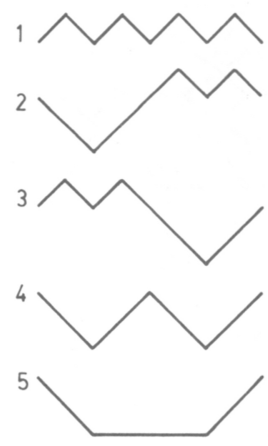

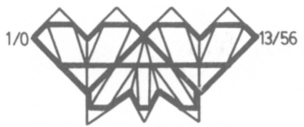

$D_{t}$

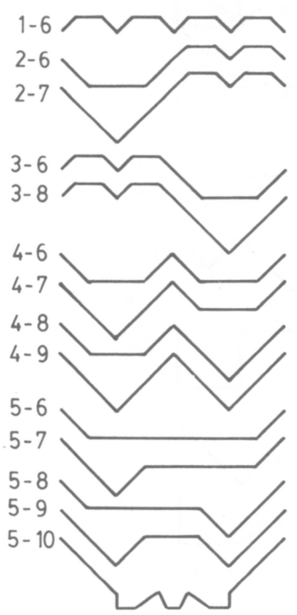

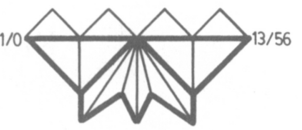

$D_{0}=D_{\infty}$

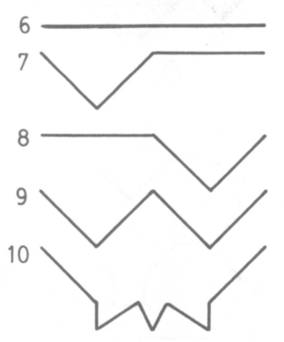

FIGURE 5.3

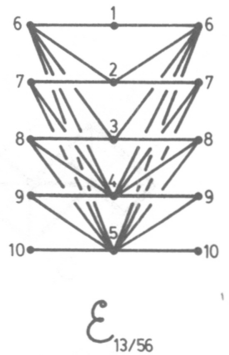

$\varepsilon_{13 / 56}$ 


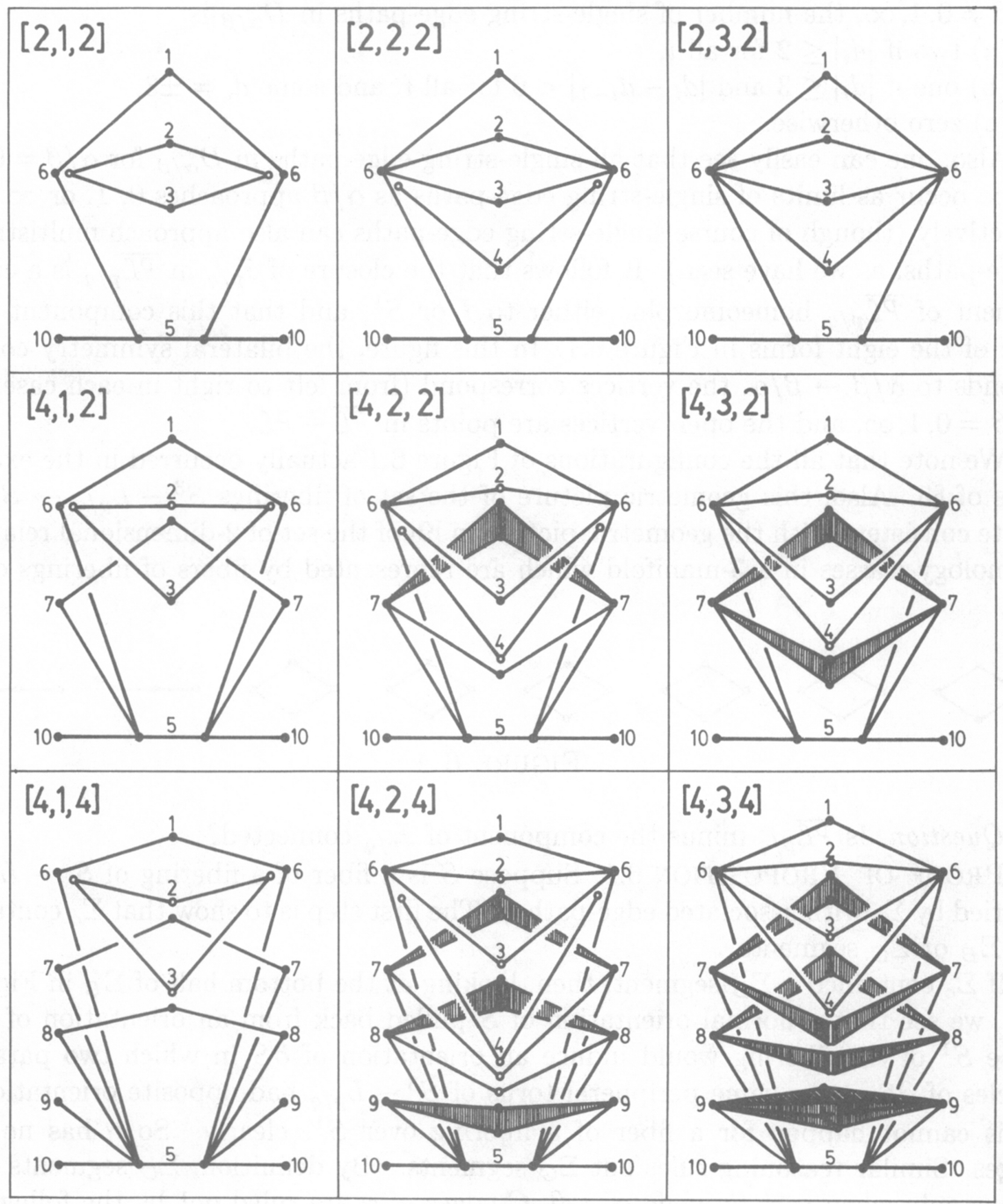

FIGURE 5.4

6. Fiberings. The isotopy classes of fibers of fiberings $S^{2}-L_{p / q} \rightarrow S^{1}$ form a subset $\mathcal{F}_{p / q}$ of $P \mathcal{L}_{p / q}$, since being a fiber is a projective property, and fibers are evidently incompressible and satisfy $(*)$ of $\S 3$. To determine $\mathcal{F}_{p / q}$ we use:

Proposition 6.1. A surface in $S^{3}-L_{p / q}$ is a fiber of a fibering $S^{3}-L_{p / q} \rightarrow S^{1}$ if and only if it is isotopic to a surface of sheet ratio $\alpha / \beta$ carried by a branched surface $\Sigma_{c}$ whose associated edge-path from $1 / 0$ to $p / q$ in $D_{\alpha / \beta}$ consists of a single string of $A$ - and $D$-edges.

For the definition of "string" see $\S 4$. As we saw there, $\Sigma_{c}$ carries a unique projective isotopy class of surfaces with given sheet ratio $\alpha / \beta$ if and only if the associated edge-path in $D_{\alpha / \beta}$ contains at most two strings. From Figure 4.1, if 
$\alpha / \beta \neq 0,1, \infty$, the number of single-string edge-paths in $D_{\alpha / \beta}$ is:

(a) two if $\left|d_{i}\right| \leq 2$ for all $i$,

(b) one if $\left|d_{i}\right| \leq 3$ and $\left|d_{i}-d_{i-1}\right|<6$ for all $i$, and some $d_{i}= \pm 3$,

(c) zero otherwise.

Also, one can easily see that all single-string edge-paths in $D_{\alpha / \beta}$ for $\alpha / \beta=0,1$, or $\infty$ occur as limits of single-string edge-paths as $\alpha / \beta$ approaches 0,1 , or $\infty$, respectively (though of course single-string edge-paths can also approach multistring edge-paths, as we have seen). It follows that the closure of $\mathcal{F}_{p / q}$ in $\overline{\mathcal{P L}}_{p / q}$ is a component of $\overline{P \mathcal{L}}_{p / q}$ homeomorphic either to $I$ or $S^{1}$, and that this component has one of the eight forms in Figure 6.1. In this figure, the bilateral symmetry corresponds to $\alpha / \beta \leftrightarrow \beta / \alpha$, the vertices correspond (from left to right in each case) to $\alpha / \beta=0,1, \infty$, and the open vertices are points in $\overline{P \mathcal{L}}-P \mathcal{L}$.

We note that all the configurations of Figure 6.1 actually occurred in the examples of $\S 5$. Also, this geometric picture of the set of fiberings $S^{3}-L_{p / q} \rightarrow S^{1}$ is quite consistent with the geometric picture in [9] of the set of 2-dimensional relative homology classes in a 3-manifold which are represented by fibers of fiberings over $S^{1}$.
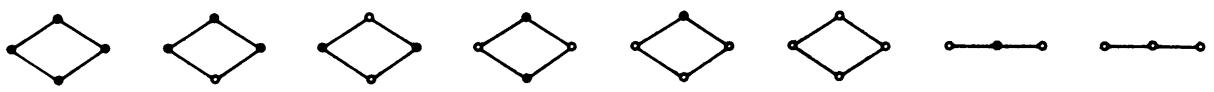

FIGURE 6.1

Question. Is $\overline{P \mathcal{L}}_{p / q}$ minus the component of $\mathcal{F}_{p / q}$ connected?

ProOF OF PROPOSITION 6.1. Suppose $S$ is a fiber of a fibering of $S^{3}-L_{p / q}$, carried by $\Sigma_{c}$ with associated edge-path $\gamma$. The first step is to show that $\Sigma_{c}$ contains no $\Sigma_{B}$ or $\Sigma_{C}$ segments.

If $\Sigma_{c}$ contained a $\Sigma_{B}$ segment, then, looking at the bottom half of $\Sigma_{B}$ in Figure 3.1 , we see that a normal orientation of $S$ pulled back from an orientation of the base $S^{1}$ of the fibering would induce an orientation of $\partial S$ in which two parallel circles of $\partial S$ in the same peripheral torus of $S^{3}-L_{p / q}$ had opposite orientations. This cannot happen for a fiber of a fibering over $S^{1}$, clearly. So $\gamma$ has no $B$ edges. Similar reasoning rules out $\Sigma_{C}$ segments. (By definition, $\Sigma_{C}$ segments can occur only when $\alpha \neq \beta$; when $\alpha=\beta, C$-edges of $\gamma$ are ruled out by the following paragraph.)

Also, we note that two successive $A$-type edges of $\gamma$ (including $C$-type edges when $\alpha=\beta$ ) must be separated by an even number of triangles in $D_{\alpha / \beta}$. Geometrically this means the union of the two corresponding $\Sigma_{A}$ segments must have an even number of half-twists in its central band (see Figure 3.2). For if the number of halftwists were odd we would reach the same forbidden orientation of $\partial S$ as before.

Next we claim that $\Sigma_{c}$ also carries a surface $S^{\prime}$ disjoint from $S$, such that $S \cup S^{\prime}$ is carried by $\Sigma_{c}$ with strictly positive weights. This concerns only a neighborhood of $\Sigma_{c}$. We can change $\gamma$ to an edge-path $\gamma^{\prime}$ (perhaps ending at a different $p / q$ ) having the same sequence of $A$ - and $D$-edges, such that $\gamma^{\prime}$ consists of a single string. The associated $\Sigma_{c^{\prime}}$ has a neighborhood homeomorphic to a neighborhood of $\Sigma_{c}$. In $\Sigma_{c^{\prime}}$, we can enlarge $S$ by adding parallel copies of itself and then deform these copies using the isotopy relations of Theorem 3.1(c) to yield a surface $S \cup S^{\prime}$ carried by $\Sigma_{c^{\prime}}$ with positive weights. Transferring $S^{\prime}$ back to $\Sigma_{c}$ gives the claim. Since $S^{\prime}$ is 
disjoint from $S$ and we are assuming $S^{3}-L_{p / q}$ fibers with fiber $S, S^{\prime}$ must also consist of fibers. So there is no loss of generality in assuming $S$ itself is carried by $\Sigma_{c}$ with positive weights. (As mentioned earlier, being a fiber is a projective property.)

Let $N$ be a neighborhood of $\Sigma_{c}$ which is fibered by intervals transverse to $\Sigma_{c}$, as in [3]. Thus $\partial N$ consists of two parts: $\partial_{h} N$, which is the closure of the set of endpoints of the fibers of $N$, and $\partial_{v} N$, which is the closure of the rest of $\partial N$. (We are regarding $S^{3}-L_{p / q}$ as an open manifold here.) Since the branching locus of $\Sigma_{c}$ contains no circles, only arcs, $\partial_{v} N$ consists of rectangles $\mathbf{R} \times I$ near these branching arcs. Replacing $S$ by two parallel copies of itself if necessary, we can isotope $S$ within $N$, staying transverse to the fibers of $N$, so that $\partial_{h} N \subset S$.

Since we assume $S$ is a fiber of a fibering $S^{3}-L_{p / q} \rightarrow S^{1}$, if we split $S^{3}-L_{p / q}$ along $S$ we get $S \times I$, with orientations chosen to make all compositions $\{s\} \times I \subset$ $S \times I \rightarrow S^{3}-L_{p / q} \rightarrow S^{1}$ for $s \in S$ orientation preserving. Each component $\mathbf{R} \times I$ of $\partial_{v} N$ has its boundary in $S \times \partial I$ and in fact we may assume $\mathbf{R} \times\{0\} \subset S \times\{0\}$ and $\mathbf{R} \times\{1\} \subset S \times\{1\}$, since the two components of $\mathbf{R} \times \partial I$ cannot both lie in $S \times\{0\}$ or in $S \times\{1\}$. (To see this, look in the peripheral tori of $S^{3}-L_{p / q}$.) So the $I$-bundle structure on $S \times I$ can be isotoped so that $\partial_{v} N$ is a union of fibers of this $I$-bundle. Hence the components of $\left(S^{3}-L_{p / q}\right)-N$ are $I$-bundles, or, in other words, the complementary components of $\Sigma_{c}$ are $I$-bundles, with fibers meeting $\Sigma_{c}$ transversely.

To see that this implies $\gamma$ consists of a single string, there are four possibilities to consider for a complementary component $X$ of $\Sigma_{c}$ formed by two consecutive $\Sigma_{A}$ or $\Sigma_{D}$ segments of $\Sigma_{c}$.

(1) Two $\Sigma_{A}$ segments. Figure 6.2(a) shows the special case that the two $A$-edges of $\gamma$ are separated by two triangles of $D_{\alpha / \beta}$, so that one of the vertical bands in the union of the two $\Sigma_{A}$ segments has two half-twists. In general, if the two $A$ edges of $\gamma$ are separated by $m$ triangles of $D_{\alpha / \beta}$, then there are $m$ half-twists. We have already seen that $m$ is even. Topologically, $X$ is a solid torus containing two distinguished circles consisting of the four arcs of $L$ alternating with four arcs of "cusp" points $C$ of $\Sigma_{c}$. See Figure 6.2(a) for the case $m=2$. If $X$ is an $I$-bundle with fibers transverse to $\Sigma_{c}$, then it must be an annulus $A$ cross $I$, with $\partial A \times I$ pinched to $\partial A=(L \cap X) \cup C \subset \partial X$. A meridian disc of the solid torus $X$ meets $L \cup C$ in $m$ points. In the given $I$-bundle structure on $X$, one can choose a meridian disc which is the sub- $I$-bundle lying over an arc of $A$ joining the two components of $\partial A$. Hence $m=2$. Conversely, if $m=2, X$ clearly has an $I$-bundle structure transverse to $\Sigma_{c}$.

(2) A $\Sigma_{A}$ and a $\Sigma_{D}$ segment. The only choice here, up to level-preserving diffeomorphism, is shown in Figure 6.2(b). In this case $X$ is a ball with a distinguished circle of six arcs of $L$ alternating with six arcs of "cusp" points $C$. Straightening out this circle to be the equator, one sees the $I$-bundle structure on $X$.

(3) Two $\Sigma_{D}$ segments with $\alpha / \beta \neq 0, \infty$. This is quite similar to case (2); see Figure 6.2(c).

(4) Two $\Sigma_{D}$ segments with $\alpha / \beta=0$ or $\infty$, say $\alpha / \beta=\infty$. Then $X$ is a ball $B$ minus two $\operatorname{arcs} a$ and $b$ of $L$ which run through its interior. Two other $\operatorname{arcs}$ of $L$ together with two $\operatorname{arcs}$ of cusp points $C$ form a circle $\sigma$ in $\partial B$. The two $\operatorname{arcs} a$ and 


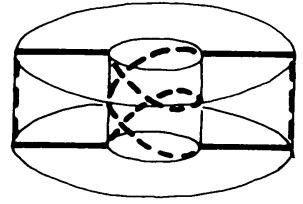

(a) $A A$

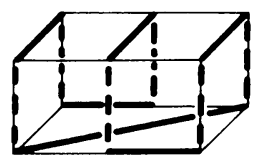

(b) $A D$

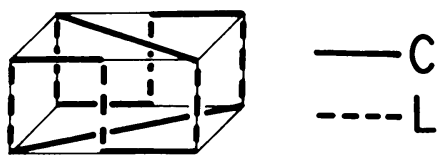

(c) DD

FIGURE 6.2

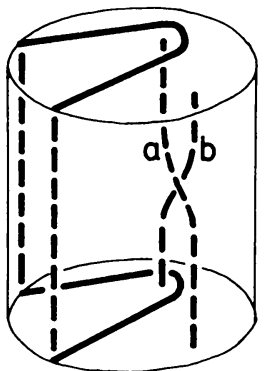

(a) $m=1$

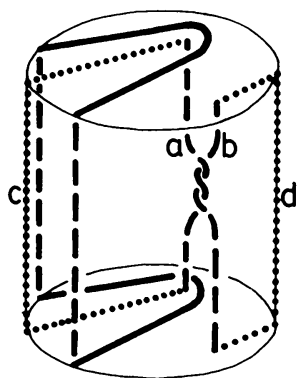

(b) $m=3$

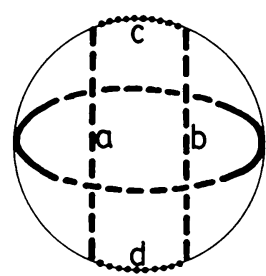

(c)

FIGURE 6.3

$b$ have $m$ half-twists, where $m$ is the number of $B$-edges of $D_{\infty}$ between the two given $D$-edges. See Figure 6.3.

The assumed fibering of $X$ extends over the ball $B$ by making $a$ and $b$ fibers. This fibering is isomorphic to the standard model in Figure 6.3(c), where the fibers are vertical segments. There is a rectangle $R \subset B-\sigma$ which is a union of fibers, $\partial R$ consisting of $a \cup b$ plus two $\operatorname{arcs} c$ and $d$ in $\partial B-\sigma$. Referring to Figure 6.3(b), we see that $\partial R$ is knotted in $B$ unless $m=1$, contradicting the existence of $R \subset B$. (Note that the two arcs of $R$ in $\partial B-\sigma$ are uniquely determined up to isotopy, so $\partial R$ is well defined independent of $R$.) Conversely, when $m=1$ it is obvious that $X$ has an $I$-bundle structure with fibers meeting $\Sigma_{c}$ transversely, since the configuration of Figure 6.3(a) is isotopic to that in Figure 6.3(c).

This completes the proof that $\gamma$ is a single string if $S$, carried by $\Sigma_{c}$, is a fiber. For the converse, if $\gamma$ is a single string, the orientable $I$-fibering of the neighborhood $N$ of $\Sigma_{c}$ extends (orientably) over the complementary components of $N$; we have already seen this except for the two components created by the initial and final $\Sigma_{A}$ (or $\Sigma_{D}$ if $\alpha / \beta=0$ or $\infty$ ) segments of $\Sigma_{c}$, where the fibering is even easier to see. Thus if $S$ is carried by $\Sigma_{c}$ with positive weights, then $S^{3}-L_{p / q}$ split along $S$ is $S \times I$, so $S$ is a fiber of a fibering $S^{3}-L_{p / q} \rightarrow S^{1}$.

7. Proof of Theorem 3.1. Let $S \subset S^{3}-L$ be a compact orientable incompressible surface with boundary on $L=L_{p / q} \subset S^{2} \times I \subset S^{3}$. We suppose $S$ has no peripheral components. This implies in particular that $S$ is $\partial$-incompressible and (by [6]) that $\partial S \neq \varnothing$. We may isotope $S$ so that:

(i) Each component of $\partial S$ is either a meridian of $L$ in $S^{2} \times(0,1)$, or is transverse to all meridians of $L$.

(ii) $S$ is transverse to $S^{2} \times \partial I$ and lies in $S^{2} \times I$ near $L \cap\left(S^{2} \times \partial I\right)$. 


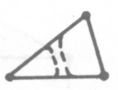

(a)

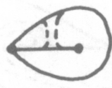

(b)

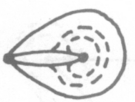

(c)

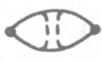

(d)

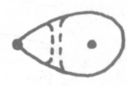

(e)

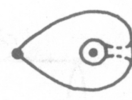

(f)

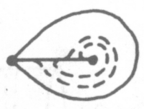

(g)

FIGURE 7.1

(iii) The projection $S \cap\left(S^{2} \times I\right) \rightarrow I$ is a Morse function with all its critical points in the interior of $S$.

A transverse intersection $S \cap S_{r}^{2}, S_{r}^{2}=S^{2} \times r$, for $0<r<1$, can contain no arcs which are peripheral in $S_{r}^{2}-L$, in view of (i) and the $\partial$-incompressibility of $S$. Thus, ignoring any circles of $S \cap S_{r}^{2}$, the arcs of $S \cap S_{r}^{2}$ determine a point $\lambda_{r}$ in the diagram $D_{\alpha / \beta}$ of $\S 1$, for the appropriate ratio $\alpha / \beta$ (which is independent of $r$ ). The case $\alpha=\beta$ was covered completely in [6], so we need consider only the cases $\alpha \neq \beta$.

As $r$ varies from 0 to 1 , the point $\lambda_{r} \in D_{\alpha / \beta}$ can change only at critical levels of the projection $S \cap\left(S^{2} \times I\right) \rightarrow I$, in fact, only at saddles. A saddle where $\lambda_{r}$ changes we call an essential saddle. So we obtain a finite sequence of $\lambda_{r}$ 's, say $\lambda_{0}, \ldots, \lambda_{k}$, with $\lambda_{i+1} \neq \lambda_{i}$ for all $i$. By (ii), $\lambda_{0}$ is the vertex $1 / 0$ of $D_{\alpha / \beta}$ and $\lambda_{k}$ is the vertex $p / q$.

Each $\lambda_{i}$ must lie in the 1-skeleton of $D_{\alpha / \beta}$. For if some $\lambda_{i}$ is in the interior of a 2-cell of $D_{\alpha / \beta}$, consider the possible positions for the $i$ th essential saddle. It is easy to see that passing this saddle produces either a peripheral level arc of $S$ (Figures 7.1(a)-(f)) or a nonorientable surface (Figure 7.1(g)).

Two successive $\lambda_{i}$ 's must lie in a common edge of $D_{\alpha / \beta}$. This is because the two curve systems $S \cap S_{r}^{2}$ just above and below a saddle, when projected to the same level $S^{2}$, can be isotoped to be disjoint. (This uses orientability of $S$ to give a normal direction in which to push one system off the other.)

By the same argument used in the proof of Lemma 2 of [6], we can isotope $S$ to lie in $S^{2} \times I$ and have all its critical points essential saddles (and also still satisfy (i)-(iii) above).

The possibilities (up to level-preserving isotopy) for an essential saddle corresponding to a segment $\left\langle\lambda_{i}, \lambda_{i+1}\right\rangle$ on an $A-, B-, C$-, or $D$-type edge of $D_{\alpha / \beta}$ are shown in Figure 7.2. In each case there is a dual picture, not drawn, corresponding to reflecting $S^{2} \times I$ across $S^{2} \times\{1 / 2\}$. Note that the pair $\lambda_{i}, \lambda_{i+1}$ determines the essential saddle uniquely (up to isotopy) in the cases of $B$ - and $C$-edges, and up to a 2 -fold ambiguity for $A$ - and $D$-edges with $\alpha \beta \neq 0$. For the $D$-edge case with $\alpha \beta=0$ there is a 4 -fold ambiguity, but in view of the meridional incompressibility condition $(*)$ it is easy to isotope the first and second pictures here to the third and fourth pictures, respectively. Thus we can reduce to only a 2 -fold ambiguity in this case. We remark further that if we allow also orientation-reversing diffeomorphisms of $S^{2}$, the two saddles of each ambiguous pair in Figure 7.2 become equivalent.

LEMMA 7.1. The $\lambda$-sequence $\lambda_{0}, \ldots, \lambda_{k}$ traces out a minimal edge-path in $D_{\alpha / \beta}$, i.e.,

(a) No pair of successive segments $\left\langle\lambda_{i}, \lambda_{i+1}\right\rangle$ and $\left\langle\lambda_{i+1}, \lambda_{i+2}\right\rangle$ lie on two different edges of the same triangle or quadrilateral of $D_{\alpha / \beta}$.

(b) $\lambda_{i+2} \neq \lambda_{i}$ for each $i$. 


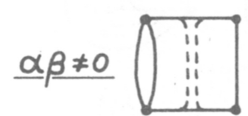

A

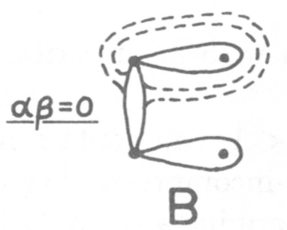

B

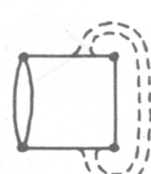

A

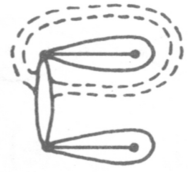

B

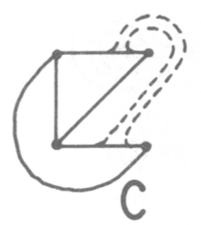

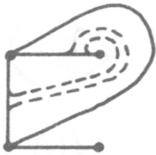

D

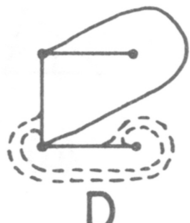

D

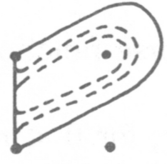

D

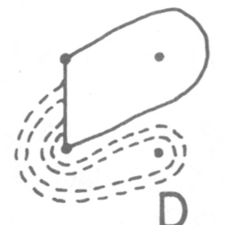

D

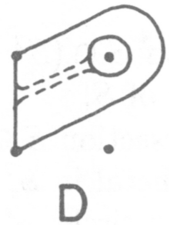

D

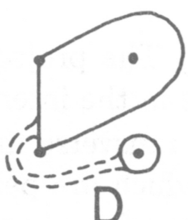

FIGURE 7.2

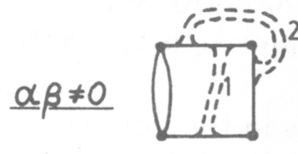

AA

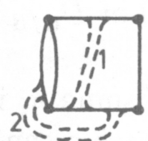

AA

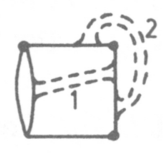

AC

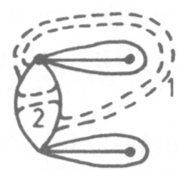

BB

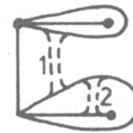

$\mathrm{BD}$

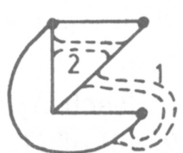

CD

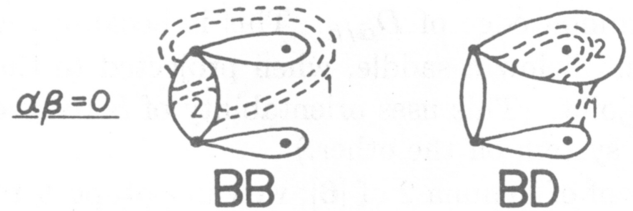

FIGURE 7.3

PROOF. (a) The essentially distinct possibilities for successive essential saddles corresponding to segments $\left\langle\lambda_{i}, \lambda_{i+1}\right\rangle$ and $\left\langle\lambda_{i+1}, \lambda_{i+2}\right\rangle$ lying on different edges of one polygon of $D_{\alpha / \beta}$ are listed in Figure 7.3. In each case, reversing the levels of the two saddles produces a peripheral level arc of $S$, a situation we have previously ruled out.

(b) Here the possibilities for $\lambda_{i+2}=\lambda_{i}$ are shown in Figure 7.4. As in the proof of (a) we see what the effect of interchanging the levels of the two saddles is. In cases $A A_{1}, A A_{4}, B B_{1}, B B_{2}, C C, D D_{1}, B B_{3}, B B_{4}, D D_{4}$ a trivial level circle results, a situation we have already seen how to simplify. In the remaining cases let $i$ be minimal such that $\lambda_{i+2}=\lambda_{i}$. In cases $A A_{2}, A A_{5}, D D_{2}, D D_{5}$, reversing the saddles yields $\lambda_{i+1}=\lambda_{i-1}$, while in the other cases $A A_{3}, A A_{6}, D D_{3}, D D_{6}$, the saddle reversal leaves $\lambda_{i}$ and $\lambda_{i+2}$ unchanged but changes $\lambda_{i+1}$ to a point on an edge which is two polygons away. This change of $\lambda_{i+1}$ to a point on an edge two polygons away can be performed at will, in particular to move $\lambda_{i+1}$ to an edge 


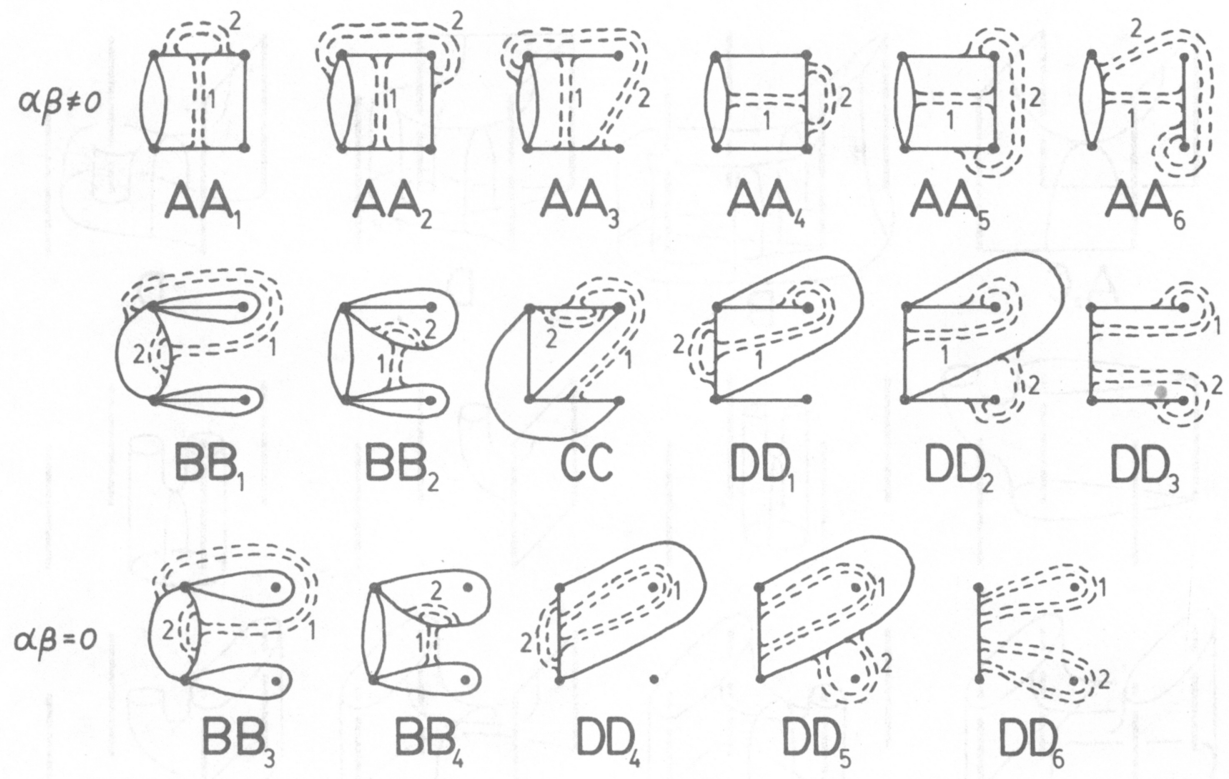

FIGURE 7.4

nearer $\lambda_{i-1}$. Repeating this, we eventually have either $\lambda_{i+1}$ on the edge adjacent to the one containing $\lambda_{i-1}$, but this was already ruled out, or $\lambda_{i+1}=\lambda_{i-1}$. Thus, by induction on $i$ we are reduced to $\lambda_{i+2}=\lambda_{i}$ with $i=0$. Here we use the fact that the two possible positions for the initial saddle changing $\lambda_{0}=1 / 0$ to $\lambda_{1}$ are isotopic across the ball in $S^{3}-S^{2} \times(0,1)$ bounded by $S_{0}^{2}$, to reduce to case $A A_{4}$.

It follows immediately from this lemma that $S$ is carried by one of the branched surfaces $\Sigma_{c}$ corresponding to a minimal edge path in $D_{\alpha / \beta}$. This is one half of Theorem 3.1(a).

For the rest of Theorem 3.1 we are free to replace a surface by the boundary of a tubular neighborhood of itself, thereby doubling $\alpha$ and $\beta$. (For part (c), note that the isotopy relations in question are simple pushes across trivial $I$-bundles in the complement of $\Sigma_{c}$, as we saw in $\S 6$.)

Consider an isotopy $S_{t}$ of a surface $S=S_{0}$ carried by some $\Sigma_{c}$, such that condition (i) at the beginning of this section holds for all $t$. Generically, the projection $S^{2} \times I \rightarrow I$ restricted to $S_{t}$ will have only nondegenerate critical points, all on distinct levels, except for two types of isolated phenomena:

(I) A pair of nondegenerate critical points of adjacent indices is introduced or cancelled in a level containing no other critical points.

(II) Two nondegenerate critical points interchange levels.

We claim that the following conditions which hold for $S_{0}$ hold also for each $S_{t}$ :

(a) No transverse intersection $S_{t} \cap S_{r}^{2}$ contains an arc which is peripheral in $S_{r}^{2}-L$.

(b) Each circle of a transverse intersection $S_{t} \cap S_{r}^{2}$ which is peripheral in $S_{r}^{2}-L$ bounds a punctured disc on $S_{t}$, the puncture being formed by the disc meeting $L$ transversely. 

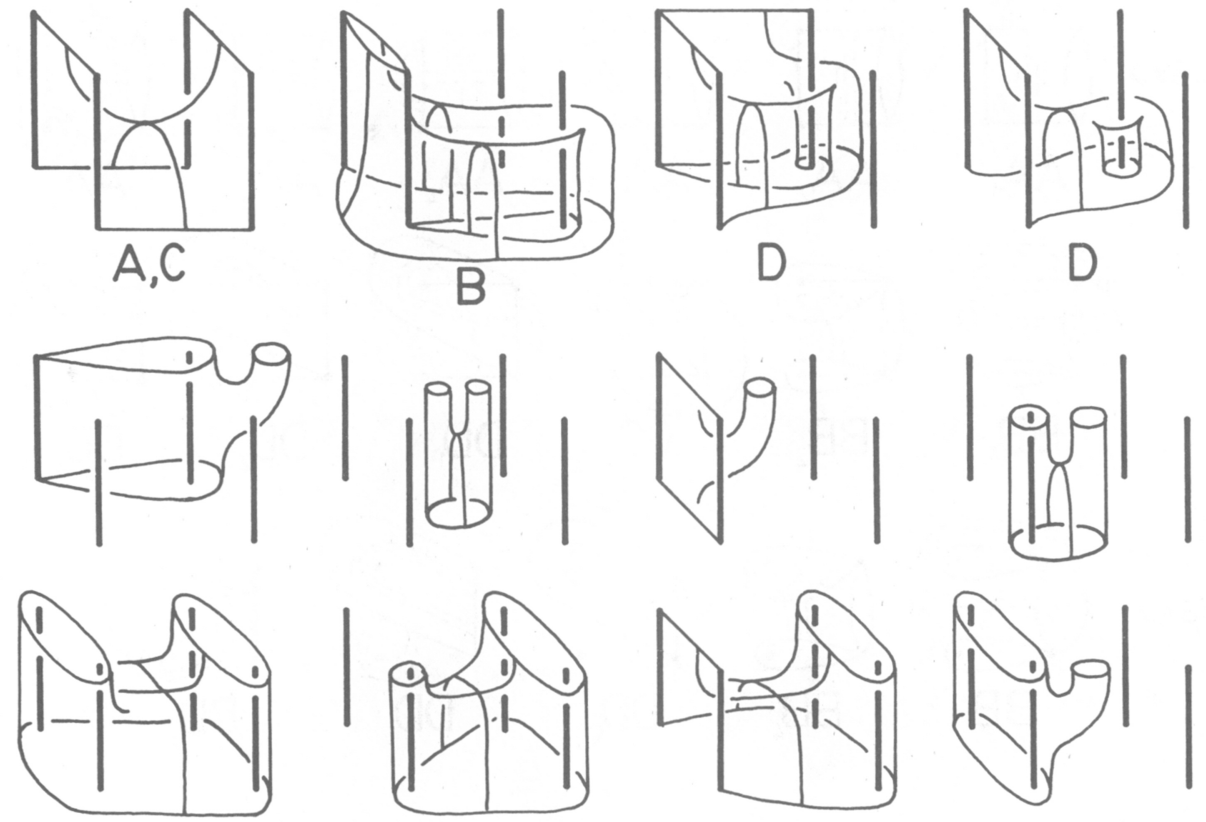

FIGURE 7.5
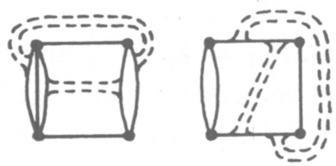

$\mathrm{AA}_{1}$

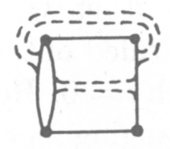

$\mathrm{AD}$

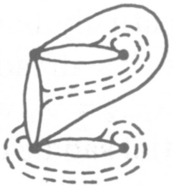

$\mathrm{DD}_{1}$

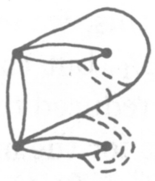

$\mathrm{DD}_{2}$

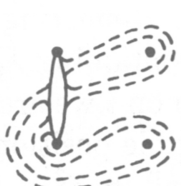

$\mathrm{DD}_{3}$

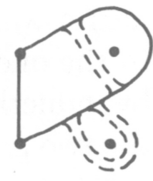

$\mathrm{DD}_{4}$

FIGURE 7.6

(c) Each circle of a transverse intersection $S_{t} \cap S_{r}^{2}$ which is nonperipheral in $S_{r}^{2}-L$ bounds a disc on $S_{t}$. Further, the number of such circles which do not bound discs in $S_{r}^{2}-L$ is zero in levels $S_{r}^{2}$ between essential saddles of $S_{t}$ and varies monotonically with $r$ above or below all the levels $S_{r}^{2}$ containing essential saddles of $S_{t}$.

(d) The $\lambda$-sequence of $S_{t}$ is the same as that of $S_{0}$ (which is minimal).

Assuming this claim, incompressibility of $S$ follows easily: If $D$ is a candidate for a compressing disc for $S$, we can isotope a small subdisc $D_{0}$ of $D$ to lie in a level $S_{r}^{2}$. Then let $S_{t}$ be the restriction to $S$ of an ambient isotopy of $S^{3}(\mathrm{rel} L)$ which shrinks $D$ to $D_{0}$. At the end of this isotopy, $S_{1} \cap S_{r}^{2}$ contains the circle $\partial D_{0}$, which is trivial in $S_{r}^{2}-L$. So by (c), $\partial D_{0}$ bounds a disc on $S_{1}$, hence $\partial D$ bounded a disc on $S$. Verification of $(*)$ is similar, using (b).

The claim that (a)-(d) are preserved during the isotopy $S_{t}$ is proved inductively, the induction step being to consider what happens at the phenomena (I) and (II). Clearly, only the case of two saddles interchanging levels is nontrivial. Each of the two saddles has one of the forms shown in Figure 7.5, modulo level-preserving 

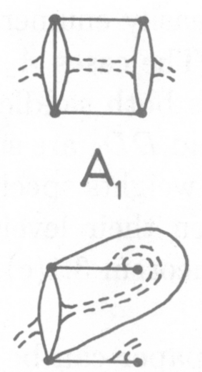

$D_{1}$

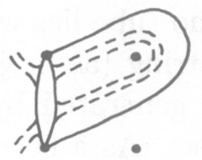

$\mathrm{D}_{4}$

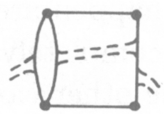

$\mathrm{A}_{2}$

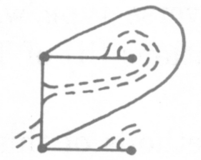

$\mathrm{D}_{2}$

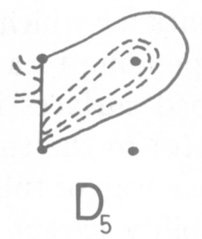

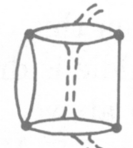

$\mathrm{A}_{3}$

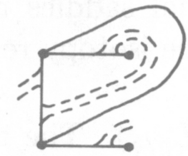

$\mathrm{D}_{3}$

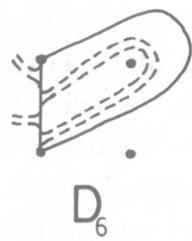

FIGURE 7.7

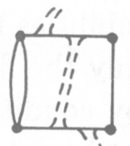

$\mathrm{A}_{4}$
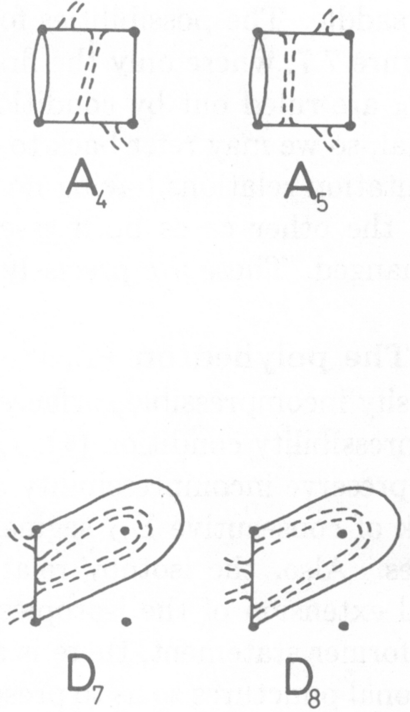

diffeomorphism and turning the picture upside down. (The four vertical arcs in each picture are the four strands of $L$.) The first four cases of Figure 7.5 are essential saddles, the rest are inessential. The cases when both saddles are essential are listed in Figure 7.6; in these cases it is evident that reversing the heights of the saddles preserves (a)-(d). In the other cases, consider $S_{t}^{\prime}$, the part of $S_{t}$ lying between a level $S_{r_{0}}$ just above the upper saddle and a level $S_{r_{1}}$ just below the lower saddle, with its horizontal boundary circles capped off by the (punctured) discs they bound in $S_{t}$ (before interchanging the heights of the saddles). The components of $S_{t}^{\prime}$ are discs, with possibly one puncture at $L$, as is evident from Figure 7.5. From this it follows that (b) and (c) are preserved when the heights of the two saddles are interchanged. Moreover, any arc of $S_{t}^{\prime} \cap S^{2} \times\left[r_{0}, r_{1}\right]$ which is peripheral in $S^{2} \times\left[r_{0}, r_{1}\right]$ splits off a disc from $S_{t}^{\prime}$. As observed at the beginning of this section, if this peripheral arc were in a level $S_{r}^{2}$, condition (i) would be violated. So (a) is preserved. That the $\lambda$-sequence is preserved is clear if both saddles are inessential. If one saddle is essential before the interchange, this saddle changing $\lambda_{i}$ to $\lambda_{i+1}$, then after the interchange this must remain true. For otherwise both saddles after the interchange would be essential, but we cannot get from $\lambda_{i}$ to $\lambda_{i+1}$ by two essential saddles, with fixed $\alpha$ and $\beta$. (We may assume $\alpha+\beta$ is large, as mentioned before.)

This completes the proof of 3.1(a). The proof of 3.1(b) is immediate from the invariance of minimal $\lambda$-sequences, property (d) above. For 3.1(c), let both ends $S_{0}$ and $S_{1}$ of the generic isotopy $S_{t}$ be carried by $\Sigma_{c}$. Their common $\lambda$-sequence determines $S_{0}$ and $S_{1}$ up to a 2 -fold ambiguity for each $A$ - or $D$-edge essential saddle, so it suffices to see if the isotopy $S_{t}$ can reverse the type of an essential $A$ or $D$-edge saddle. For the initial or final essential saddle the type can be reversed by pushing the saddle across either ball of $S^{3}-S^{2} \times(0,1)$. This is relation (i) of Theorem $3.1(\mathrm{c})$. The only other thing to check is whether interchanging the height of an essential saddle with the height of another saddle can reverse the type 
of the saddle. The possibilities for when this could happen are easily enumerated; see Figure 7.7, where only the first saddle is drawn completely. The cases $A_{5}, D_{7}$, and $D_{8}$ are ruled out by condition (i) above. In the other cases both saddles are essential, so we may refer back to Figure 7.6. Here $A A_{1}, D D_{1}$, and $D D_{3}$ are simple commutation relations having no effect on the branched surface weights specifying $S$. In the other cases both essential saddles reverse type when their levels are interchanged. These are precisely the isotopy relations (ii) of Theorem 3.1(c).

8. The polyhedron $P \mathcal{L}\left(S^{3}-L_{p / q}\right)$. The methods of this paper can be used to classify incompressible surfaces in $S^{3}-L_{p / q}$ which do not satisfy the meridional incompressibility condition $(*)$. One finds that the sequences of peripheral tubings which preserve incompressibility are exactly the ones for which no tube lies within a block of consecutive $\Sigma_{D}$ segments of $\Sigma_{c}$ corresponding to a string (as in $\S 4$ ) of $D$-edges. Also, the isotopy relation for such tubed surfaces is generated by the natural extension of the isotopies in Theorem 3.1(c) to the tubes. As a corollary of the former statement, there is always at least one way of tubing together all the meridional punctures so as to preserve incompressibility, except when the associated edge-path is a single string, i.e., the surface is a fiber of a fibering over $S^{1}$. (If the number of punctures is odd, first replace the surface by two parallel copies of itself.)

Allowing surfaces not satisfying $(*)$, but still incompressible, $\partial$-incompressible, and without peripheral torus components, has the effect of enlarging $P \mathcal{L}_{p / q}$ to a polyhedron $P \mathcal{L}\left(S^{3}-L_{p / q}\right)$ by adjoining cells to the subcomplex of $P \mathcal{L}_{p / q}$ where $\alpha$ or $\beta$ is zero. These cells correspond to certain branched surfaces obtained by modifying the branched surfaces $\Sigma_{C}$ which meet one component of $L_{p / q}$ in meridians, modifying these branched surfaces so that they carry the various admissible peripheral tubes. Details are left to the reader. It can be checked that meridional surgery leads to a deformation retraction of $P \mathcal{L}\left(S^{3}-L_{p / q}\right)$ onto $P \mathcal{L}_{p / q}$. Most of the interest and subtlety in $P \mathcal{L}\left(S^{3}-L_{p / q}\right)$ seems to reside in the subpolyhedron $P \mathcal{L}_{p / q}$.

As a simple example, for the Whitehead link $L_{3 / 8}=L_{[2,1,2]}, P \mathcal{L}\left(S^{3}-L_{3 / 8}\right)$ is obtained from $P \mathcal{L}_{3 / 8}$ by attaching a 2 -simplex by one of its vertices at each of the two vertices of $P \mathcal{L}_{3 / 8}$ labelled "6" in Figure 5.4.

9. Boundary slopes. Another question which could be investigated in detail is to determine which systems of curves on the two peripheral tori $T_{1}$ and $T_{2}$ of $S^{3}-L_{p / q}$ bound incompressible surfaces (satisfying $(*)$ ) in $S^{3}-L_{p / q}$. That is, one seeks to compute the boundary map $\mathcal{P L}\left(S^{3}-L_{p / q}\right) \rightarrow \mathcal{P L}\left(T_{1} \cup T_{2}\right)=\mathbf{R} P^{1} * \mathbf{R} P^{1}=$ $S^{3}$. According to [4], the image is one-dimensional. It can be shown that this map factors

$$
\mathcal{P L}\left(S^{3}-L_{p / q}\right) \rightarrow \tilde{\mathcal{E}}_{p / q} \rightarrow \operatorname{P\mathcal {L}}\left(T_{1} \cup T_{2}\right),
$$

the first map being the natural projection and the second map being (projective) linear on simplices of $\tilde{\mathcal{E}}_{p / q}$. An explicit formula for this boundary-slope map $\tilde{\mathcal{E}}_{p / q} \rightarrow$ $\mathcal{P L}\left(T_{1} \cup T_{2}\right)$ in terms of the combinatorics of minimal edge-paths in $D_{0}$ and $D_{1}$ is probably computable, but it appears the formula may be somewhat complicated, so we shall not pursue the matter here. 


\section{REFERENCES}

1. J. H. Conway, An enumeration of knots and links, and some of their algebraic properties, Computational Problems in Abstract Algebra, Pergamon, New York and Oxford, 1970, pp. 329-358.

2. W. Floyd and A. Hatcher, Curves on the 4-punctured sphere: 31 charts, unpublished manuscript.

3. W. Floyd and U. Oertel, Incompressible surfaces via branched surfaces, Topology 23 (1984), 117-125.

4. A. Hatcher, On the boundary curves of incompressible surfaces, Pacific J. Math. 99 (1982), 373-377.

5. __ Projective lamination spaces for 3-manifolds, in preparation.

6. A. Hatcher and W. Thurston, Incompressible surfaces in 2-bridge knot complements, Invent. Math. 79 (1985), 225-246.

7. U. Oertel, Incompressible branched surfaces, Invent. Math. 76 (1984), 385-410.

8. W. Thurston, On the geometry and dynamics of diffeomorphisms of surfaces, preprint.

9. __ A norm for the homology of 3-manifolds, Mem. Amer. Math. Soc., vol. 59, no. 339, 1986, pp. $99-130$.

10. H. Schubert, Knoten mit zwei Brücken, Math. Z. 65 (1956), 133-170.

11. L. Siebenmann, Exercises sur les noeuds rationnels, xeroxed notes from Orsay, 1975.

12. U. Oertel, Measured laminations in 3-manifolds, MSRI preprint \#11112-85, 1985.

Department of Mathematics, Virginia Polytechnic Institute and State UNIVERSITY, BLACKSBURG, VIRGINIA 24061

Department of Mathematics, Cornell University, IthaCA, NeW YoRK 14853 